\title{
Yoga Research: A Narrative Review
}

\author{
Tiffany Field* \\ Department of Pediatrics, University of Miami, USA
}

Submission: November 16, 2019; Published: January 03, 2020

*Corresponding author: Tiffany Field, Department of Pediatrics, Touch Research Institute, University of Miami, Miller School of Medicine, Fielding Graduate University, USA

\begin{abstract}
This paper is a narrative review on yoga research published during the last few years (2016-2019). The review includes summaries of papers on prevalence of yoga and the use of yoga for physical and mental health. Most of the studies in the current literature have addressed yoga effects on mental and physical health conditions in adults, although some studies have focused on prenatal, pediatric and aging samples. The literature on adult mental health conditions includes stress, depression, anxiety and PTSD as well as substance disorders. The physical conditions include tinnitus, obesity and eating disorders and many studies that are focused on pain syndromes including neck pain, low back pain and knee pain and disease-related pain (e.g. fibromyalgia, diabetes, inflammatory bowel disease, multiple sclerosis and cancer).

The aging conditions treated by yoga include cardiovascular disease, stroke, Parkinson's and dementia Studies on biomarkers are suggestive of underlying mechanisms for yoga effects including increased heart rate variability, increased connectivity based on fMRI data and reduced cortisol and pro-inflammatory cells. Yoga has typically been more effective than inactive control and waitlist control conditions, although it has not always been more effective than active comparison groups (i.e. other forms of exercise). Having established the physical and mental health benefits of yoga makes it ethically questionable to assign participants to inactive control groups. Although further research is needed, the studies reviewed here highlight the therapeutic effects of yoga, a practice that is increasingly being called yoga therapy..
\end{abstract}

Keywords: Yoga; Physical; Mental health; Pro-inflammatory cells; Exercise; Yoga therapy

\section{Introduction}

This paper is a review of yoga research including empirical studies, systematic reviews and meta-analyses that have been published over the last few years to update our last review Flied [1]. The term yoga was entered as a title term into PubMed, and the selection criteria were empirical studies (pre-post, randomized controlled) in which standard treatment, waitlist or other active treatment (exercise) groups were compared to yoga groups (of different styles). Exclusion criteria were case studies, qualitative studies, small sample pilot studies, studies in which assessors were not "blind" to group assignment and non-English papers. Following these criteria, this review includes summaries of 174 papers selected from a total of 933 papers that appeared on PubMed (231 for 2016, 101 for 2017, 361 for 2018 and 240 for 2019).

These papers have focused on demographics/prevalence of yoga and the use of yoga for physical and mental health conditions. Most of the studies in the current literature have addressed yoga effects on these conditions in adults, although some studies have focused on prenatal, pediatric and aging samples. The literature on adult yoga includes mental health problems, e.g. stress, depression, anxiety and PTSD as well as substance use disorders.
The physical condition studies include obesity and eating disorders and pain syndromes including neck pain, low back pain, knee pain and disease-related pain (e.g. fibromyalgia, diabetes, inflammatory bowel disease, multiple sclerosis and cancer).

The aging conditions found in the recent yoga literature include cardiovascular disease, stroke, Parkinson's and dementia. Biomarker studies have documented increased heart rate variability as well as increased connectivity based on fMRI data and reduced cortisol and pro-inflammatory cells following yoga. This review is accordingly divided into sections on the prevalence of yoga, on prenatal yoga, and yoga with pediatric (child and adolescent) and with adult samples. The adult section is divided into subsections on stress, mental health and physical conditions. The physical conditions section is further subdivided into sections on obesity, eating disorders and pain syndromes including musculoskeletal and disease-related pain. These are followed by summaries of studies on aging conditions and on special samples including veterans and prison inmates. A section on biomarkers includes physiological and biochemical measures including heart rate variability, brain connectivity, cortisol and pro-inflammatory cells. Methodological limitations of this literature and future research directions are discussed at the conclusion of the paper. 


\section{Prevalence, Demographics and Safety of Yoga Practice}

\section{Surveys of practitioners}

Surveys published in the last few years highlight the growth of yoga. In the most recent of these on 34,525 individuals, yoga had increased from 6\% in 2002 to $15 \%$ in 2017 Wang Li \& Gaylord [2]. Only $7 \%$ of the users were referred by physicians, and the top three medical conditions for yoga use included acute and chronic pain, arthritis and depression. In another survey $(\mathrm{N}=116,404)$, lifetime prevalence of yoga use was 13\% (31 million users), while one-year prevalence was 9\% (21 million) Cramer \& Zhang [3]. In this study, $70 \%$ of users practiced for general wellness or disease prevention, $66 \%$ to increase energy and $50 \%$ to improve immune function. For more specific conditions, back pain was reported by $20 \%$, stress by $6 \%$ and arthritis by $6 \%$. In this survey, lifetime practitioners were more often female, younger, non-Hispanic white, college-educated, higher earners, living in the West and in better health.

In still another survey, although yoga use had increased significantly over a ten-year period, adherence failed to increase and use for specific health problems including back pain declined as well as referral-driven yoga Patwardhan \& Lloyd [4]. The decrease in use for health problems like back pain was surprising given that the research on yoga for health problems including back pain had increased during the same time period. A lesser prevalence of $7 \%$ has been reported for Australian women $(\mathrm{N}=1925)$ Lauche \& Cramer [5]. In this survey, predictors of practicing yoga were being married or in a relationship and having greater health and more education. Not surprisingly, obesity and diabetes decreased the likelihood of using yoga. In contrast, in a U.S. study, being in a relationship was negatively correlated with practicing yoga although obesity was again negatively correlated with practicing yoga $(\mathrm{N}=34,525)$ Cramer \& Lauche [6]. In this sample, being a vegetarian was also associated with practicing yoga.

\section{Surveys of yoga teachers/therapists}

Surveys have also been conducted with yoga teachers/ therapists in this recent literature. In a survey of North American yoga instructors $(\mathrm{N}=367)$, for example, 91\% were female and $88 \%$ were between the years of 40 and 70 Sullivan \& Moonaz [7]. Most yoga instructors conducted therapeutic yoga classes (91\%) with more than half delivering 1-10 therapeutic classes per month. The conditions that were seen most frequently were back/neck pain (77\%), joint pain/stiffness (67\%) and anxiety (77\%). These therapists were spending on average eight hours a week in their clinical practice, with $41 \%$ earning an annual income of less than $\$ 10,000$ from yoga therapy. For example, in a survey on 968 Iyengar yoga instructors, $81 \%$ reported teaching therapeutic yoga either in groups or privately Ross \& Wallen [8]. Although they noted that most practitioners came for help for over 54 different health conditions, the majority attended yoga classes for musculoskeletal conditions and injuries followed by pregnancy and hypertension. Almost the entire sample reported that pain brought practitioners to yoga most frequently. The instructors also noted that they modified the poses in yoga classes for students according to their health conditions and symptoms.

\section{Surveys on adverse events/injuries}

The prevalence of adverse events/injuries has also been the focus of many surveys. In a systematic review of nine observational studies on 9029 yoga practitioners and 9903 non-practitioners, the incidence of adverse events happening during yoga classes was $23 \%$, although serious adverse events occurred in only $2 \%$ of the practitioners Cramer \& Lauche [9]. The most common injury was musculoskeletal including sprains and strains. The yoga practitioners and non-practitioners had comparable risks of falling and fall-related injuries. In a cross-sectional survey of German yoga practitioners, the incidence of injuries was similar at 29\% Cramer \& Lauche [10]. And, again, most adverse events were musculoskeletal that most frequently occurred during hand, shoulder and head stands. Although the most common yoga styles were Ashtanga and traditional Hatha, the greatest rate of injuries occurred during power yoga. In another study, the poses that were most commonly associated with injuries involved hyperflexion and hyperextension of the spine which, again, would likely happen most frequently during shoulder and head stands Lee \& Sinaki [11]. Although the more typical poses involve plantar flexion and external rotation of the ankle joints, the ankle joints have not been as involved in injuries Mears \& Mannen et al. [12]. Independent of injuries, the incidence rate for pain caused by yoga was $11 \%$ in a sample of 354 practitioners Campo \& Pappas [13]. In contrast to the preceding studies, a meta-analysis has suggested that yoga as compared to non-yoga exercise was not associated with increased injuries. And, although this result was based on only three randomized controlled trials, the data from these trials are consistent with previous meta-analyses Cramer, Lauche et al. [14].

Because yoga exercises have been associated with joint problems, some have suggested that yoga may be potentially dangerous for joint health Lauche \& Cramer [15]. In this study, this research group assessed 9151 women 62-67 years-old who were participating in the Australian Longitudinal Study on Women's Health. Multiple regression modeling suggested that $30 \%$ reported regular problems with stiff painful joints including $16 \%$ for problems with the feet, $18 \%$ with knees, $12 \%$ with hips and $15 \%$ with shoulders. Practicing yoga for these joint problems highlighted the safety of yoga.

Fortunately, yoga has not involved injuries during the traditional forms. They tend to occur only during very difficult poses. The safety of yoga also applies to prenatal yoga for both the mother and the fetus inasmuch as pre-conception and prenatal women are not usually practicing shoulder stands and head stands. 


\section{Prenatal Yoga}

Table 1: Prenatal yoga effects and first authors.

\begin{tabular}{|c|c|}
\hline Effects & First authors \\
\hline$<$ less fertility problem stress & Kirca \\
\hline$<$ stress \& anxiety & Darbandi \\
\hline$>$ fetal outcomes & \\
\hline$<$ prenatal depression & Ng \\
\hline$<$ labor pain & \\
\hline$<$ labor induction & \\
\hline$<$ caesarean section & \\
\hline$<$ time in labor & Chethana \\
\hline$<$ time in labor & \\
\hline$<$ pain tolerance & \\
\hline$<$ labor induction & \\
\hline$<$ caesarean section & \\
\hline
\end{tabular}

Prenatal yoga programs have been used not only for problems like infertility but also for continuing exercise throughout pregnancy. And, ultimately, prenatal yoga has effectively facilitated reproduction, reduced labor pain and facilitated delivery outcomes (Table 1) see table 1 for list of prenatal yoga effects. Infertility. A couple studies have been conducted on lowering stress levels and assisting reproduction in infertile women. In a randomized controlled study on 128 women who were registered for in vitro fertilization and were receiving treatment for infertility, the yoga program was conducted twice a week for six weeks Kirca, \& Pasinlioglu [16]. Based on the Fertility Problem Stress Scale, the difference between the pre-and posttest for the experimental group was greater than for the control group. In a literature review based on 87 studies on infertility, yoga reduced pain as well as stress, depression and anxiety and yoga improved fetal outcomes Darbandi \& Sadeghi [17].

Table 2: Pediatric yoga effects and first authors.

\begin{tabular}{|c|c|}
\hline Effects & First authors \\
\hline \multicolumn{2}{|c|}{ Children } \\
\hline > visual attention & Jarraya \\
\hline \multicolumn{2}{|l|}{$<$ hyperactivity } \\
\hline$>$ concentration, memory & Gulati \\
\hline \multicolumn{2}{|l|}{$>$ motor speed, self esteem } \\
\hline$<$ stress & Nanthakumar \\
\hline
\end{tabular}

\section{Prenatal depression}

In a meta-analysis on yoga-based interventions for prenatal depression, 8 clinical studies were systematically reviewed Ng Venkatanarayanan \& Lim, et al. [18]. Although statistically significant reductions were noted in depression scores following the yoga interventions, most of the trials included participants with only mild depression and relatively small samples. Nonetheless, these results are consistent with previous studies on the positive effects of yoga on prenatal depression [1].

\section{Labor and delivery}

Two studies have appeared in the recent literature on pain alleviation by yoga during labor and the positive birth outcomes that have resulted from yoga. In one of these studies, the yoga group attended classes three times a week starting at 26 weeks gestation, suggesting the positive effects of a longer duration participants reported lower pain intensity at 3-4 cm dilation and at two hours after the first and second measurements. The mothers who received yoga also had a decreased frequency of labor induction, a lower incidence of Caesarean section, and a shorter duration of the second and third stages of labor. In a similar study, integrated yoga was provided for 30-minute sessions every two weeks from the 30th to the 39th week of gestational age Chethana \& Saxena [20]. In this study, there was a shorter stage of the late first stage of labor, greater pain tolerance, a lesser requirement of labor induction and analgesics and significantly more vaginal deliveries than Caesarean sections. In addition, the number of low birth weight babies was also significantly lower for the yoga group. These data are consistent with an earlier study showing that prenatal yoga contributed to greater gestational age and higher birthweight infants Field \& Hernandez [21].

\section{Pediatric Yoga}

In the recent literature on yoga, a number of studies have featured children, although surprisingly few studies have involved adolescents. The pediatric studies have primarily focused on enhancing cognitive and social competence in non-clinical samples and in reducing symptoms in clinical samples including children with anxiety, autism, obesity, asthma, cystic fibrosis, muscular dystrophy and epilepsy (Table 2) see Table 2 for list of pediatric yoga effects. program Jahdi \& Ghaseminejad et al. [19]. In this study, the yoga 


\begin{tabular}{|c|c|}
\hline$<$ anxiety & \\
\hline > body image & Halliwell \\
\hline \multicolumn{2}{|l|}{$>$ positive mood states } \\
\hline > smooth gait & Hainsworth \\
\hline \multicolumn{2}{|l|}{ > emotional well-being } \\
\hline$<$ asthma episodes & Pandy \\
\hline \multicolumn{2}{|l|}{$<$ drug intake } \\
\hline$<$ anxiety \& pain & McNamara \\
\hline \multicolumn{2}{|l|}{ > respiration } \\
\hline$<$ epileptic seizures & Kanhera \\
\hline \multicolumn{2}{|l|}{$<$ abnormal EEGs } \\
\hline > heartrate variability & Vidyashree \\
\hline$>$ heartrate variability & Pradnya \\
\hline \multicolumn{2}{|c|}{ Adolescents } \\
\hline > social competence & Kwasky \\
\hline \multicolumn{2}{|l|}{ > flexibility } \\
\hline > social competence & Choukse \\
\hline \multicolumn{2}{|l|}{ empathy } \\
\hline \multicolumn{2}{|l|}{ >altruism } \\
\hline \multicolumn{2}{|l|}{$>$ relationships with parents } \\
\hline$>$ executive function & Vhavle \\
\hline \multicolumn{2}{|l|}{ > attention } \\
\hline \multicolumn{2}{|l|}{$>$ working memory } \\
\hline$<$ inflammatory bowel symptoms & Arruda \\
\hline < abdominal pain & Evans \\
\hline > sleep & \\
\hline
\end{tabular}

\section{Children}

Cognitive and social competence: In a study on the youngest practitioners, kindergarten children practiced Hatha yoga twice per week for 30 minutes for 12 weeks Jarraya \& Engel [22]. By the end of the study, the children in the yoga group were showing improved visual attention and visual-motor behavior as well as decreased inattention and hyperactivity as compared to a group who engaged in physical education. In a sample of 10 -year-old children, yoga was practiced for 60 minutes per day for four and a half months Gulati \& Balkrishna [23]. By the end of the study, the children showed improved attention, concentration, memory, motor speed and self-esteem. In addition, the teachers rated the children as having better behavior with their peers and their teachers. Unfortunately, this was a pre-post design study rather than a randomized controlled trial.

Stress and anxiety: Stress and anxiety in grade school children was the focus of a recent narrative review on 8 yoga studies that included asanas (postures), pranayama (breathing exercises), dharana (concentration) and dhyana (meditation) Nanthakumar
[24]. Despite the limitations noted in these studies including small sample sizes and heterogeneity of the yoga practice, the consistent findings suggested that yoga reduced stress and anxiety in children.

Autism: In a study on 50 children with autism spectrum disorder, the children were randomized to a group that received three months of yoga training or a control group Vidyashree \& Partheeban [25]. At the end of the training, a significant increase was noted in heart rate variability in the yoga group, suggesting enhanced attentiveness since increased heart rate variability is associated with increased attention.

Muscular Dystrophy: Given that cardiac disorder is the second most common cause of death in children with muscular dystrophy, physical therapy is typically prescribed to maintain their cardioautonomic function Pradnya \& Meghana et al. [26]. In this study, 124 children with muscular dystrophy were randomized to receive physical therapy alone or physical therapy plus yoga. Heart rate variability was measured at three-month intervals across one year. Increased heart rate variability was noted across 
nine months for the physical therapy group but across twelve months for the physical therapy plus yoga group.

Body image: In a large sample study on 344 British children, a four-week yoga intervention was provided for 9-11-year-old children who were randomly assigned to a yoga or to a physical education control group Halliwell \& Slater [27]. As in many studies that compared yoga with other forms of physical exercise, both groups had an improved body image, and both had an increase in positive mood states.

Obesity: Pediatric obesity has notably impacted the gait and emotional well-being of children. In a pre-post study, a 2-month Iyengar yoga intervention (one-hour classes twice per week) led to significant improvements in several gait parameters including hip, knee and ankle motion as well as improved emotional wellbeing Hainsworth \& Tran et al. [28]. Surprisingly, there were no changes in physical activity or body weight. A longer duration yoga intervention may be required to increase physical activity and reduce weight.

Asthma: In a one-year long yoga program, children with chronic asthma experienced fewer asthma episodes, and their scores were higher on an asthma control test Pandya [29]. In addition, they had reduced drug intake as compared to a control group. The strongest predictor of these positive results was self-practice.

Cystic fibrosis: Cystic fibrosis is the most common genetic, lifelimiting condition among children McNamara \& Thygeson et al. [30]. It is often accompanied by depression, anxiety and pain. In a pre-post study, 20 children practiced a list of 30 yoga asanas during six one-on-one session over a 10-week period [30]. Anxiety and pain scores decreased, and respiration improved following this protocol.

Epilepsy: Epilepsy typically begins in childhood, but although yoga has been studied in adults with epilepsy, only one yoga study has focused on children with epilepsy. In this recent randomized controlled trial, 20 children with a diagnosis of epilepsy who were on antiepileptic drugs were given 10 one-hour yoga sessions Kanhera \& Mukherjee [31]. At the end of six months, none of the children in the yoga group had seizures while three of the children in the control group had seizures. In addition, at the end of the treatment period only one abnormal EEG was noted in the yoga group whereas seven of the 10 children in the control group had abnormal EEGs.

\section{Adolescents}

\section{Social competence}

In one of the few studies on yoga in adolescents, young adolescents practiced yoga twice weekly for two months Kwasky \& Serowoky [32]. The adolescents showed significant improvement on social scores and on waist circumference and flexibility. The authors suggested that social improvement probably related to belonging to a group, namely a yoga group. Social competence has also improved following a seven-day yoga intervention that was given in a residential setting Choukse [33]. In this pre-post study, improvements were also noted on empathy, altruism and relationships with parents. The researchers expected that these psychosocial improvements would also affect academic performance, although that was not measured.

\section{Cognitive function}

Cognitive function was studied in a randomized controlled trial on 802 students who were randomly assigned to receive one-hour daily yoga training or physical exercise for two months Vhavle \& Manjunath [34]. The results suggested that both yoga and physical exercise improved executive function, attention and working memory. Once again, yoga and exercise protocols had similar effects.

\section{Cardio-respiratory function}

In a study sensing cardio-respiratory function, adolescents were randomized to receive one hour of daily yoga training or physical exercise over a period of two months Satish \& Shreeganesh et al. [35]. In this study, $\mathrm{VO}_{2}$ (the maximum amount of oxygen utilized during exercise) was assessed as a measure of aerobic endurance or cardiovascular fitness. There was a greater improvement in $\mathrm{VO}_{2}$ in the yoga group possibly because the physical exercise was too strenuous for that group.

\section{Irritable bowel syndrome}

Surprisingly, irritable bowel syndrome was the only clinical condition that appeared on adolescents in this recent yoga literature. Irritable bowel syndrome is increasingly common in adolescents and is debilitating for recurrent abdominal pain and altered bowel habits. In a study on adolescents' irritable bowel syndrome, the adolescents were given three 60 -minute in-person yoga classes at three times over an eight-week period and three 30-minute yoga videos per week Arruda \& Yeh [36]. The adolescents experienced less pain and other physical symptoms of irritable bowel syndrome as well as reduced stress, although they had difficulty completing the yoga videos due to time limitations and competing priorities. In a study on a six-week Iyengar yoga intervention with adolescents aged 14 to 17 years, only half the adolescents responded successfully to the yoga including their experiencing reduced abdominal pain and improved sleep Evans \& Zeltzer [37]. The responders reported having experienced benefits early in the treatment period and that their parents were supportive and committed to the intervention. Notably, both the responders and the non-responders talked about the importance of home practice to sustain these benefits, although non-compliance might be an issue with adolescents.

\section{Adult Yoga Research}

Most of the research on yoga effects has been conducted with adults which reflects the greater prevalence of adult yoga 
practitioners. Recent research on yoga effects can be categorized as yoga for mental health and physical health conditions. Unlike the earlier published research that focused primarily on healthy practitioners versus non-practitioners, the more recent research has more frequently involved adults with mental health or physical health conditions [1].

\section{Mental health conditions}

Table 3: Adult yoga effects on mental health conditions and first authors.

\begin{tabular}{|c|c|}
\hline Effects & First authors \\
\hline$>$ mindfulness & Kishida \\
\hline \multicolumn{2}{|l|}{$>$ self-compassion } \\
\hline$>$ mindfulness & Trent \\
\hline \multicolumn{2}{|l|}{$>$ positive affect } \\
\hline \multicolumn{2}{|l|}{$<$ stress } \\
\hline$>$ mental well-being & Telles, Tulloch, Domingues \\
\hline $\begin{array}{c}<\text { stress, anxiety, depression and } \\
\text { insomnia }\end{array}$ & Maddux \\
\hline$<$ stress and job burnout & Cocchiara \\
\hline$<$ stress & Puerto Valencia \\
\hline$<$ depression, stress \& anxiety & Shohani \\
\hline$<$ depression, anxiety \& pain disorder & Saeed \\
\hline$<$ depression \& anxiety & Vollbehr \\
\hline$<$ depression \& anxiety & Nguyen-Feng \\
\hline$<$ anxiety disorder & Tolahunase, Bukar \\
\hline$<$ PTSD symptoms & Cramer, Kelly \\
\hline$<$ nicotine dependence & Kuppili, Jeffries \\
\hline
\end{tabular}

Psychological conditions that have improved following yoga include mindfulness, psychological well-being and stress which are among the only few studies that appear in the recent literature on healthy practitioners. The mental health problems that have been assessed for yoga effects on clinical samples include depression, anxiety, post-traumatic stress disorder and substance use disorders see (Table 3) for list of yoga effects on mental health conditions.

\section{Mindfulness}

Increased mindfulness has been noted following yoga in at least two studies on healthy practitioners. In one of these, 104 community-dwelling yoga practitioners who practiced at least once a week were recruited for a three-week daily diary study Kishida [38]. On days that the yoga practitioners practiced more yoga as compared to a usual care group, they experienced greater mindfulness and self-compassion. Then in the second study on yoga effects on mindfulness, a five-day program was delivered at the Kripalu Center for Yoga and Health which included five hours per day of yoga, meditation, lectures and experiential activities Trent \& Dusek [39]. Relative to the controls, the yoga group experienced greater mindfulness as well as more positive affect and less stress both immediately after and two months after the end of the program. This study is not only confounded by the different activities in the five hour per day program but also because the participants were not randomly assigned to groups.

Greater mindfulness was associated with positive affect in both previous studies. And mindfuness might explain the greater usage of positive terms in a study that involved a lexical analysis of 3000 words collected before and after yoga classes Blades, \& MacFadyen [40]. This study suggested that more words connoted positivity following yoga classes, although there was no increase in thought processes involving creativity.

Mental well-being. Mental well-being was the primary dependent measure in a cross-sectional study on yogaexperienced versus yoga-naïve individuals Telles \& Balkrishna [41]. The results suggested that the yoga-experienced group had significantly greater mental well-being which in turn was correlated with the number of months that yoga had been practiced.

In a meta-analysis on yoga and mental well-being, 12 trials of high methodological quality including 752 adults age $60+$ years suggested a positive effect of yoga on mental well-being Tulloch \& Tiedemann [42]. In a similar systematic review, the effects of yoga poses were reviewed across 14 studies Domingues [43]. The most common positive mental health outcomes included mindfulness and well-being

\section{Stress}

Moderate to high stress employees were randomly assigned to 16 consecutive weeks of yoga or to a waitlist crossover group who did not practice yoga for eight weeks and then practiced yoga for eight weeks Maddux \& Telled [44]. Surprisingly, the groups practiced for 16 weeks showed equivalent reductions in stress, anxiety, depression and insomnia. Stress has been the focus of so many yoga studies that at least two systematic reviews were found in this recent literature. In the first of these, 11 studies on management of stress in healthcare workers met inclusion criteria Cocchiara \& Polimeni et al. [45]. Yoga not only prevented stress in healthcare workers but also reduced job burnout. In the other systematic review on yoga effects on the management of stress in the workplace, only 13 of 1343 papers met inclusion criteria Puerto Valencia \& Heinze [46]. Once again, the primary effect of yoga was the reduction of stress.

\section{Depression}

Depression has also been the focus of many yoga studies. In a quasi-experimental study with a pre-post-test design, 12 sessions of Hatha yoga practice were effective in reducing not only depression but also stress and anxiety Shobani \& Rahmati et al. [47]. The effects on all three of these problems are not surprising 
given that they are typically comorbid conditions Field \& Kuhn et al. [48]. In a review of systematic reviews and meta-analyses, yoga as a single or a complementary therapy had positive effects on depression Saeed Cunningham \& Bloch [49]. In addition, as a complementary therapy, yoga facilitated treatment of anxiety disorders, most especially panic disorder [49]. However, in a systematic review and meta-analysis on 18 studies, yoga reduced depression when compared to psychoeducation control groups but not as compared to other active control groups like exercise Vollbehr \&, Steenhuis et al. [49]. And, with respect to anxiety, yoga also had no significant effects when compared to other exercise groups. In another systematic review and quantitative synthesis, 12 articles met inclusion criteria Nguyen-Feng, \& Butler [51]. The yoga sessions ranged from two days to 16 weeks [51]. Their results showed only weak evidence for yoga reducing depression and anxiety following traumatic life experiences due to the low quality and high risk of bias studies that were included in the analysis. In the only study on major depression disorder (MDD), patients with MDD diagnoses were randomly assigned to receive yoga or drug therapy Tolahunase \& Dada [52]. Yoga was effective for those patients who were resistant to antidepressants. In another study on inpatients with anxiety disorders, Hatha yoga was offered three times per week and anxiety symptoms were significantly decreased after only two classes Bukar \& Davidson [53].

\section{Post-traumatic stress disorder (PTSD)}

Post-traumatic stress disorder has also been the focus of a sufficient number of yoga studies that at least two systematic reviews have been published recently. In one of these, seven randomized controlled trials were included Cramer \& Dobos [6]. In this meta-analysis on PTSD and yoga, low-quality evidence was found on clinically relevant effects of yoga as compared to no treatment. However, this was a small number of randomized controlled trials, and they were small samples. In another systematic review, only yoga studies that had at least one physiological variable were included Kelly \& Noggle Taylor [54]. This research group noted that although much of the literature suggests physiological mechanisms underlying yoga effects on PTSD, very few studies have actually included physiological measures. They, nonetheless, proposed a psychoneuroimmunology model on mechanisms related to activation of the hypothalamic-pituitary-adrenal axis and inflammation.

\section{Substance use disorders}

In a study that compared Hatha yoga to a wellness control condition (i.e. reading educational materials about a healthy lifestyle), craving for smoking decreased at a greater rate in the 30-minute Hatha yoga group relative to the wellness condition Jeffries \& Buckner [55]. In a narrative review on yoga for managing substance use disorders, 314 studies were found but only 16 studies fulfilled the inclusion and exclusion criteria and only 12 were randomized controlled trials Kuppili \& Balhara [56]. The majority of these studies involved yoga as management for nicotine dependence. The sample sizes of these studies surprisingly ranged from 18 to 624, and of these, the majority suggested that yoga was effective in reducing substance use as well as substance-related craving, especially for nicotine-use disorders but only for the short-term.

\section{Physical Health Conditions}

This section includes yoga research on tinnitus, obesity and eating disorders as well as yoga for musculoskeletal and diseaserelated pain. The musculoskeletal pain studies are focused on migraine, neck pain, low back pain and knee osteoarthritis. The disease-related pain conditions include fibromyalgia, diabetes, inflammatory bowel disease, premenstrual syndrome, multiple sclerosis and several forms of cancer. see (Table 4) for list of yoga effects on physical health conditions). Tinnitus. The perception of sound in the absence of an external source of sound is an increasingly prevalent condition Koksoy \& Vayisoglu [57]. In this study, the distressing "ringing in the ears" was not reduced although the stress, handicap and severity was reduced after three months of weekly yoga classes.

Table 4: Adult yoga effects on physical health conditions and first authors.

\begin{tabular}{|c|c|}
\hline Effects & First authors \\
\hline < severe tinnitus & Koksov \\
\hline $\begin{array}{c}\text { > enjoyment of physical activities in obese } \\
\text { individuals }\end{array}$ & Telles \\
\hline metabolic demand same as treadmill & Pryor \\
\hline$>$ number poses $>$ weight loss & Forseth \\
\hline$<$ eating disorder symptoms & Domingues, Karlson \\
\hline$>$ body flexibility & Kongkaew \\
\hline
\end{tabular}

\section{Physical fitness}

In a meta-analysis on randomized controlled trials on yoga for physical fitness, seven studies met inclusion criteria Kongkaew \& Meesomperm [58]. Yoga training was not only related to greater flexibility but also increased range of motion for both right shoulder extension and right shoulder abduction. Although the unique findings for the right shoulder versus the left shoulder are difficult to interpret, the measurement of flexibility and range of motion made this a unique study in the recent literature on yoga.

\section{Weight loss}

Although weight loss is presumably the goal of many who participate in yoga, weight loss is rarely measured in research on yoga. Weight loss yoga videos have been commercially available, and in one study, those videos were content analyzed for the duration of routines, the number of completed poses, the body position of each pose and the pose rate Forseth. In that study, weight loss routines featured the greatest number of poses and the fastest pose rate as compared to beginner routines and stress relief/meditation routines. Weight loss videos also included more 
standing poses and a lower percentage of seated and supine poses. Yoga has also been studied for its effect on body image NeumarkSztainer \& Rydell [59]. Participants who were greater weight were more likely to report a positive impact on their body image than those who were lower weight.

\section{Obesity}

Obesity is one of the most prevalent medical conditions and, as such, it is a research funding priority. Even though yoga is an exercise that might be expected to reduce obesity, very little research was found on obesity in this recent yoga literature. The two studies that were found showed positive effects of yoga on obesity, but neither showed effects on weight loss. In the first study, a group of Asian-Indian obese adults who were yoga-experienced were compared to a group who were yoga-naïve Telles \& Arya et al. [60]. The group who were experienced reported having a better quality of life including enjoyment of physical activities, ability to work, self-esteem and social satisfaction. In a second study, the researchers compared the metabolic demands of moderate intensity walking on a treadmill to yoga performed at various tempos Pryor \& Moore-Reed [61]. The metabolic equivalents observed in moderate (four seconds/pose) and high (three seconds/pose) tempo yoga were equivalent to treadmill walking.

\section{Eating disorders}

The paucity of yoga research on eating disorders is also surprising. In one study, adult females with bulimia were randomized to an 11-week yoga group (90-minute sessions two times per week) or a control group Karlsen \& Benum [62]. Although there were no differences at the end of the therapy period on the Eating Disorders Inventory, the yoga group had reduced Eating Disorder Examination scores that included restraint and eating concerns. The group differences were even greater at the six-month follow-up period, suggesting that the yoga participants may have continued the practice after the end of the study. In a systematic review on yoga and eating disorders, 12 studies met criteria Domingues \& Carmo [63]. Although the results across the studies were inconsistent, yoga practice was typically related to healthier eating behavior, fewer eating disorder symptoms, more positive body image and body satisfaction, suggesting that yoga practitioners may be at less risk for developing eating disorders.

\section{Musculoskeletal Pain}

Pain has been the most frequently claimed reason for practicing yoga including musculoskeletal and disease-related pain. The pain syndromes discussed under musculoskeletal pain include migraine headaches, neck pain, low back pain and knee osteoarthritis. see (Table 5) for list of yoga effects on musculoskeletal pain). Chronic pain in general has been addressed in a randomized controlled trial which primarily assessed occupational performance after an eight-week yoga practice versus usual care Schmid [64]. In this study, pain and depression were reduced and significant improvements in occupational performance scores were noted for the individuals who had been randomized to the yoga group. In a selective review, four meta-analyses were found that covered multiple pain conditions including chronic headache, neck pain, back pain, fibromyalgia, pelvic pain, irritable bowel syndrome and chronic fatigue syndrome Sutar \& Desai [65]. The meta-analyses indicated modest effectiveness of yoga for these disorders.

Table 5: Adult yoga effects on musculoskeletal pain and first authors.

\begin{tabular}{|c|c|}
\hline \multicolumn{1}{|c|}{ Effects } & First authors \\
\hline \multicolumn{2}{|c|}{ General pain } \\
\hline$<$ pain & Schmid, Sutar \\
\hline \multirow{2}{*}{$<$ pain } & Monson, Hartfield \\
\hline \multirow{2}{*}{$<$ migraine headaches } & Sharma \\
\hline \multirow{2}{*}{$<$ neck pain } & Dunleavy, Kim 2016, 2018 Cramer \\
\hline \multirow{2}{*}{$\begin{array}{c}\text { < low back pain } \\
\text { < knee pain }\end{array}$} & Telles, Kuvacic, Patil 2015, 2018 \\
\cline { 2 - 2 } & Saper, Neyaz, Whitehead, Wieland \\
\hline \multirow{2}{*}{$<$ rheumatoid arthritis pain } & Park, Chueng 2016, 2017 \\
\cline { 2 - 3 } & Mathersul, Kuntz, Kan, Wang, Lauche \\
\hline
\end{tabular}

\section{Musculoskeletal pain in general}

In a study on musculoskeletal pain in general, two yoga sessions per week (60-minute sessions) were provided for a convenience sample of dental hygiene students for 13 weeks Monson \& KrenikMatejcek [66]. The yoga group reported a significant decrease in musculoskeletal pain. In a similar study that occurred in the workplace, participants were randomly assigned to an eight-week yoga program with a six-month follow-up Hartfield \& Edwards [67]. At the six-month follow-up, the yoga group reported only having missed two workdays due to musculoskeletal pain versus the usual care comparison group that missed 43 days.

\section{Migraine headaches}

Yoga effects on migraine headaches have surprisingly been rarely researched. An exception was a study that combined yoga therapy and Ayurveda Sharma \& Ertsey [68]. Following 90 days of intervention, the therapy group showed a significant reduction in migraine symptoms including pain intensity.

\section{Neck pain}

Chronic neck pain has been experienced by roughly half of the population Allende \& Kramer [69]. Unlike the paucity of research for migraine headaches, several studies have been conducted on neck pain including a few systematic reviews and metaanalyses. In a pretest-posttest design study, university students were assigned to a yoga exercise group (one-hour sessions for two days a week for two months) Kim [70]. The yoga exercises included flexion of the neck, extension of the neck, right lateral flexion of the neck, left lateral flexion of the neck, right rotation of 
the neck and left rotation of the neck. Pain was simply measured by a $100 \mathrm{~mm}$ visual analogue scale. By the end of the two-month study, the yoga group showed significantly lower neck pain scores compared to the control group. This protocol may have been especially effective because of its focus on the region of pain, namely the neck.

In a nine-week Iyengar yoga course, participants with chronic non-specific neck pain were randomized to either the yoga group or self-directed exercise Allende \& Cramer [69]. The degree to which they benefited varied and they did not experience a consistent reduction in neck pain intensity. The sample size for this unconditional growth model study may have been too small for the complexity of the data analysis. In another neck pain study, yoga was compared with Pilates Dunleavy \& Talley et al. [71]. The pain ratings decreased significantly for both the Pilates and the yoga groups. Surprisingly, no improvements in range of motion or posture were noted for either group.

In one of the systematic reviews of randomized trials on yoga for neck pain, only three trials were identified as meeting criteria Kim [72]. Nonetheless, those studies suggested that neck pain intensity and functional disability were significantly lower in the yoga versus the control groups. In another systematic review and meta-analysis on the effects of yoga on chronic neck pain, again only three studies were found to meet criteria Cramer \& Dobos [73]. The results revealed short-term effects for neck pain intensity, neck pain-related disability and quality of life. In still a third systematic review and meta-analysis on 10 trials including 686 participants, yoga was compared with other interventions $\mathrm{Li}$ \& Yuan [74]. The results suggested that yoga had positive effects on neck pain intensity, neck pain-related functional disability, cervical range of motion and mood. As the authors suggested, however, a comprehensive summary of all the evidence was difficult due to the different types of yoga and duration of the yoga interventions as well as the different outcome measures. Chronic low back pain. Chronic low back pain affects millions of people worldwide and appears to be increasing in prevalence Chang \& Groessl [75]. And, it is the most frequently researched form of pain in the yoga literature. Chronic low back pain has also been associated with increased disability, psychological symptoms and reduced quality of life. In a feasibility study that compared 14 practices yoga led to lower back pain ratings, disability ratings and perceived stress Patil \& Subramanya [76]. This study, however, was limited by not having any treatment comparison groups or control conditions.

In another low back pain study, 62 adults with degenerative intervertebral discs were randomly assigned to yoga and control groups Telles \& Balkrishna [77]. The yoga protocol included physical postures, breathing exercises and relaxation techniques for one hour daily for 12 weeks. Although the yoga group showed a significant reduction in self-reported pain and state anxiety, they did not show MRI changes in the intervertebral discs and the vertebrae. These results are confounded by the combination of breathing exercises, postures and relaxation. In still another study on low back pain, 30 individuals were assigned to a yoga or an educational pamphlet group Kuvacic \& De Giorgio [78]. The yoga group participated in an eight-week (two days per week) program that also included education on spine anatomy/biomechanics and the management of chronic low back pain. Although the yoga group experienced significant decreases in pain, anxiety and depression, there were no group differences on disability. These results, again, are confounded by the addition of education and management to the yoga therapy.

More mixed results emerge when yoga is compared to a different form of exercise. In a study in which 88 female nurses were randomized to a yoga group or a physical exercise group, the groups were equivalent on the time spent exercising (one hour per day for five days a week for six weeks) Patil \& Patil [79]. In this case, although both groups experienced reduced pain, yoga led to greater pain reduction. In a study that compared yoga and physical therapy for chronic low back pain, participants were randomly assigned to 12 weekly yoga classes or 15 physical therapy visits or an educational book/newsletter group Saper \& Sadokova et al. [80].

The yoga and physical therapy groups had lower levels of pain medication than the education group and the reduction of back pain was maintained for both the yoga and physical therapy groups as late as one year after the treatment. This long-term effect was surprising because the positive effects would not be expected to extend beyond the treatment period. But this result may be related to the participants having continued treatment following the end of the study. In a similar exercise comparison study, patients with chronic low back pain were randomly assigned to Hatha yoga or to a conventional therapeutic exercise group Neyaz \& Wadhwa [81]. Both groups attended 35-minute weekly sessions for 12 weeks and the participants were asked to practice on non-class days at home. Once again, the two types of exercise had equivalent effects.

In a systematic review of yoga for the treatment of low back pain, most of the 12 trials that met criteria used Iyengar or Hatha forms of yoga Whitehead \& Gould Fogerite [82]. When yoga was compared to non-exercise controls, low back pain was reduced, and back-related function was improved in the yoga but not the non-exercise control groups. However, when yoga was compared to non-yoga exercise controls, there were no group differences in pain reduction and back-related function at the three-month follow-up assessments. Similarly, in another systematic review, nine trials (810 participants) of yoga compared to non-exercise controls showed pain reduction and improved back-related function for the yoga versus the non-exercise groups Wieland, et al. [83].

But when four trials (394 participants) of yoga were compared to non-yoga exercise controls, no group differences were noted in pain reduction and back-related function. In contrast, when three systematic reviews that included 10 randomized controlled trials 
were reviewed, significant effects were noted for short and longterm pain reduction and long-term effects were noted for reduced back disability in the yoga versus the control groups Goode et al. [84]. This literature could be summarized as yoga reducing low back pain when compared to non-exercise control groups but having equivalent pain reduction effects when compared to other exercise groups. This is not surprising given that both forms of exercise are stimulating pressure receptors that would lead to pain reduction via increased serotonin or reduced substance $\mathrm{P}$, although these were not measured [1]. The almost exclusive reliance on self-report in these studies limits the power of their results.

\section{Knee pain}

Knee pain is most commonly a symptom of osteoarthritis which is a highly prevalent and disabling chronic condition Cheung, Justice \& Peden-McAlpine [85]. Physical activity is a critical component of the management of osteoarthritis, and yoga has become increasingly popular for managing osteoarthritis. Because this condition is more prevalent in older adults, attempts have been made to modify yoga for that age group. For example, chair yoga has been offered in a senior center in a recent study Park, McCaffrey, Newman, Liehr \& Ouslander [86]. Older adults (N= 131) were randomly assigned to chair yoga or a health education program. Both groups met twice weekly for 45-minute sessions for two months. The chair yoga group showed greater reduction in pain interference that was surprisingly sustained for 3 months after the end of the intervention. However, although pain, gait speed and fatigue scale scores improved for the yoga group, they were not sustained after the end of the intervention. The authors reported in another publication that once the participants were no longer in group sessions for yoga, they did not continue their yoga practice McCaffrey, Park \& Newman [87]. If the yoga practice was not continued, it is not surprising that its pain-relieving effects were not sustained. If the pain relief is related to the stimulation of pressure receptors, the pain relief would not be expected to continue in the absence of that stimulation.

One of the ways that yoga researchers are attempting to sustain the benefits is by having participants engage in home practice sessions usually by following sessions on DVD. For example, in a randomized controlled trial with three arms, Hatha yoga was compared to aerobics and an education control group Cheung, Wyman, Bronas, McCarthy, Rudser, et al. [88]. The two exercise groups met for eight weekly 45- minute classes with the expectation that the participants would then engage in home practice sessions for 2 to 4 days a week. At the end of the study, the Hatha yoga group had a greater improvement in pain, anxiety and fear of falling than the other two groups. The more positive effects for yoga than aerobics may relate to the aerobics being too strenuous and/or the lesser compliance with the home aerobics than the home yoga. Tele-yoga (real-time interactive video conferencing) has also been an effective home therapy for knee pain Mathersul, Mahoney \& Bayley [89].
In another randomized controlled trial, 31 women with knee osteoarthritis were assigned to one of three groups including a yoga exercise, a traditional exercise and a no exercise attention control group (a meditation group) Kuntz, Chopp-Hurley, Brenneman, Karampatos, Wiebenga, et al. [90] The participants were asked to attend three one-hour group classes each week for three months. The yoga group experienced greater improvement in pain and physical function and the traditional exercise group showed greater improvement in flexor strength. No changes were noted in the meditation group.Several reviews have been published in the last few years on the use of yoga with knee osteoarthritis. In one of the first of these, most of the studies that were reviewed showed a clinically significant reduction in pain especially research that focused on poses (for example, the Iyengar studies) as opposed to those that had integrated protocols including poses, breathing and meditation exercises [1]. In a focused review, Iyengar and Hatha yoga were the most commonly used types of yoga Cheung, Park \& Wyman [91]. The frequency of the yoga sessions ranged from once a week to six days a week and the duration of the sessions ranged from 45 to 90 minutes per session for 6 to 12 weeks. The data from these 12 studies on 59 participants with knee osteoarthritis suggested reductions in pain, stiffness and swelling. However, the physical function results were inconclusive because of the variety of outcome measures that were used.

In a systematic review on six studies involving 372 patients with knee osteoarthritis, the most common yoga protocol was 40-90 minutes per session lasting for at least eight weeks Kan, Zhang, Yang \& Wang [92]. In these studies, the pain relief and improvement in function could be seen as early as two weeks into the interventions. In a systematic review and meta-analysis on 13 clinical trials involving 1557 patients, yoga was effective for reducing arthritic symptoms including pain and for promoting physical function Wang, Lu, Wang, Jiang, Rao, Wang et al. [93]. In another systematic review and meta-analysis on nine trials including 640 individuals with knee osteoarthritis, yoga was effective for reducing pain and stiffness and improving function as compared to both exercise and non-exercise control groups Lauche [5]. These results were surprising given that several other yoga studies have shown equivalent effects of yoga and other forms of exercise.

\section{Rheumatoid Arthritis}

Pain reduction has also been the focus of yoga studies on patients with rheumatoid arthritis. In a recent survey on the prevalence of yoga among 398 patients with rheumatoid arthritis, $88 \%$ were females, $66 \%$ were white, the mean age was 62 years, the mean duration of the disease was 25 years and only $11 \%$ participated in yoga Greysen [94]. The most common styles were Vinyasa, Hatha, Iyengar and restorative yoga which were mostly practiced in group settings. The yoga participants were significantly more likely to work full-time, less likely to be unable to work due to disability and had better physical function. 
In a study on the management of pain and sleep in adults with rheumatoid arthritis, the participants were randomized to either eight weekly 75-minute yoga classes or a usual care control group Ward [95]. The retention rates were 100 percent for yoga and $92 \%$ for usual care at the 12 -week follow-up assessment. Pain reduction was greater in the yoga group and, surprisingly, more than half the yoga group participants reported continuing yoga at home after the end of the study.

In an important biomarker study, 72 rheumatoid arthritis patients were randomly assigned to a yoga group that also received anti-rheumatic drug therapy or a group that received the drug therapy alone Gautam 2019. After eight weeks of yoga, a significant decrease was noted in the severity of rheumatoid arthritis as evidenced by a reduction in the levels of various systemic inflammatory markers as well as in the erythrocyte sedimentation rate (a faster than normal rate that may indicate inflammation). In addition, significant reductions in disability and depression were noted in the yoga group.

\section{Disease-Related Pain}

The disease-related pain conditions reviewed here include fibromyalgia, diabetes, inflammatory bowel disease, premenstrual syndrome, multiple sclerosis and several forms of cancer. All of these have benefited from yoga practice/therapy. see (Table 6) for list of yoga effects on disease-related pain.

Table 6: Adult yoga effects on disease-related pain and first authors.

\begin{tabular}{|c|c|}
\hline Effects & First authors \\
\hline$<$ pain, <stress & Koksoy \\
\hline$<$ pain,> sleep & Carson \\
\hline$<$ pain, > strength & Hegde \\
\hline \multicolumn{2}{|c|}{$>$ antioxidants in diabetes } \\
\hline $\begin{array}{c}<\text { pain, glucose, cholesterol, triglycerides } \\
\text { in diabetes }\end{array}$ & Mondal \\
\hline$<$ pain \& anxiety & Sharma, Schumann \\
\hline$<$ pain, > sleep in premenstrual syndrome & Ghaffarilaleh \\
\hline$<$ menstrual pain & Yonglitthipagon, Yang \\
\hline$<$ menstrual pain, $>$ sleep & McGovern \\
\hline$<$ pelvic pain & Saxena, Kinser \\
\hline $\begin{array}{c}\text { < pain, fatigue, depression in multiple } \\
\text { sclerosis, Kahraman }\end{array}$ & Hasanpour Dehkordi \\
\hline$<$ shoulder \& arm pain in breast cancer & Eyigor \\
\hline $\begin{array}{c}<\text { pain, fatigue, } \& \text { sleep disturbance in } \\
\text { breast cancer }\end{array}$ & Cramer 2017 \\
\hline$<$ pain \& fatigue in multiple cancers & Danhauer \\
\hline$<$ pain, fatigue, anxiety \& depression & Kuloor \\
\hline
\end{tabular}

\section{Fibromyalgia}

In a three-month study on yoga with fibromyalgia patients, changes were noted in the processing of pain (Carson 2016).
In this sample, significant improvements were noted in pain tolerance and pressure pain threshold at the post-treatment assessment. Fibromyalgia symptoms and functional deficits also improved including physical tests of strength and balance as well as pain coping strategies. These effects may derive from enhanced sleep following yoga and, in turn, a reduction in substance $P$ that causes pain, as has been noted in other studies [1].

\section{Diabetes}

Although pain is not usually the focus of yoga for diabetes studies, pain has been reduced in type 2 diabetes Schmid, Alter, Malcom, Grimm, Klinedinst, Marchant et al. [96]. In this eightweek study, yoga was more effective than usual care not only for reducing pain but also for increasing upper and lower extremity strength. In another study on type II diabetes, participants were randomly assigned to a yoga group or a stretching exercise group for three months (Hegde 2019). Again, not only was pain reduced but the levels of glutathione (antioxidant) were increased in the yoga group. The yoga included both postures and breathing exercises so it is again not clear which aspect of yoga was contributing to these effects.

In still another diabetes study (three sessions per week for 12 weeks), participants were randomly assigned to a yoga or control group Mondal, Kundu \& Saha [97]. In this study, pain decreased but also decreases were noted in fasting plasma glucose, postprandial blood sugar, total cholesterol, triglycerides, low density lipoprotein and very low-density lipoprotein as well as increased high density lipoprotein. Again, the yoga practice included both poses and breathing exercises. In a systematic review and meta-analysis on eight studies including 842 participants, yoga as compared to exercise had greater effects on glycemic control including fasting blood glucose and postprandial blood glucose as well as glycosylated hemoglobin and body mass index Jayawardena, Ranasinghe, Chathuranga, Atapattu \& Misra [95].

\section{Inflammatory bowel disease}

Inflammatory bowel disease (IBD) (also known as irritable bowel syndrome) is a chronic illness characterized by inflammation in the gastrointestinal tract that results in abdominal pain, cramping, diarrhea and bloody stools Sharma, Poojary, Dwivedi \& Deepak [98]. A few studies in this recent literature have documented the positive effects of yoga on these symptoms. In one study, 100 IBD patients were randomly assigned to yoga (eight weeks of physical postures, breathing and meditation exercises one hour per day) or a control group that received standard medical therapy alone Sharma et al., [98]. At the end of the eight-week intervention, a significant decrease was noted in pain as well as state and trait anxiety levels. In a systematic review on six randomized controlled trials with a total of 273 patients, yoga was noted to decrease pain as well as other irritable bowel symptoms and anxiety Schumann, Anheyer, Lauche, Dobos, Langhorst \& Cramer [99]. 


\section{Premenstrual syndrome and menstrual pain}

Women with premenstrual syndrome (also called dysmenorrhea) typically complain of pain and associated problems including sleep disturbances and depression. In a study on premenstrual syndrome, 62 women were randomly assigned to a yoga or a control group Ghaffarilaleh, Ghaffarilaleh, Sanamno, Kamalifard \& Alibaf [100]. The yoga group received three sessions per week lasting 60-minutes for a 10-week period. Premenstrual pain was reduced as well as sleeping problems including the quality of sleep, sleep latency and sleep efficiency. In a later paper by the same group, the same yoga practice by the same sample resulted in significantly lower diastolic blood pressure and depression Ghaffarilaleh, Ghaffarilaleh, Sanamno \& Kamalifard [101].

\section{Yoga has also reduced menstrual pain}

In one study on 64 participants, 91\% reported experiencing menstrual pain Tsai [102]. After a 12-week yoga intervention, the participants reported decreased pain, use of analgesics, abdominal swelling, breast tenderness and cold sweats. The reduction in menstrual pain was correlated with the other effects. In a later paper by the same group, the same yoga practice by the same sample resulted in significantly lower diastolic blood pressure and depression Ghaffarilaleh, Ghaffarilaleh, Sanamno \& Kamalifard [101]. In another study on menstrual pain, a yoga group practiced for 60-minutes once per week for 3 months and similarly showed decreased menstrual pain Yang \& Kim [103]. Menstrua pain has been reduced even when yoga was practiced for only 30-minute sessions, twice per week for 12 weeks Yonglitthipagon, Muansiangsai, Wongkhumngern, Donpunha, Chanavirut, et al. [104]. In a systematic review on 14 randomized controlled trials on yoga for menstrual pain $(\mathrm{N}=1409)$, several improvements were noted including reduced pain, improved sleep, reduced negative feelings and improved social relationships McGovern, \& Cheung [105].

\section{Pelvic pain}

Pelvic pain is such a common problem that a special questionnaire has been developed for its assessment called the Impact of Pelvic Pain Questionnaire and at least one systematic review has been published on the recent literature. In a randomized controlled study, 60 female patients with chronic pelvic pain were assigned to a yoga group that received poses, breathing exercises and meditation and were compared to a treatment as usual control group Saena, Gupta, Shankar, Jain \& Saxena [106].After eight weeks of yoga, pain intensity was significantly decreased. In a twice weekly Iyengar yoga class, participants were asked to practice yoga at home for one hour a week for six weeks and to keep a daily log of pelvic pain Huang, Rowen, Abercrombie, Subak, Schembri, et al. [107].Once again, the women showed improved scores on the Impact of Pelvic Pain Questionnaire. In a systematic review on a surprisingly large number of yoga for pelvic pain studies ( $\mathrm{N}=1435)$, only 15 met criteria Kinser, Pauli, Jallo,
Shall, Karst, Hoekstra et al. [108].Overall, these studies showed decreased pain and pain-related symptoms including decreased depression and stress following yoga.

\section{Multiple sclerosis}

Multiple sclerosis (MS) is an autoimmune disease that involves the brain and spinal cord and multiple symptoms including pain, fatigue, depression and cognitive impairment Hasanpour Dehkordi [109]. In a randomized controlled trial, 90 patients with multiple sclerosis were assigned to a yoga, aerobics exercise or control group Hasanpour Dekhordi [110]. The groups met for 40 minutes for three sessions per week for three months. At the end of the treatment period, pain and fatigue were relieved in those patients who were in the yoga and exercise groups. When the yoga group was compared to the non-exercise group in a subsequent paper, the yoga group experienced a greater decrease in pain and fatigue Hasanpour Dehkordi, Jivad \& Solati [109]. In a study by another research group, a six-month yoga training was given once a week for one hour Kahraman, Ozdogar, Yigit, Hosgel, Mehdiyev, et al. [111]. In this study, participants with MS were compared to healthy participants, but the completion rates were only $44 \%$ for those with MS and 18\% for those healthy participants and only those with MS appeared to experience a decrease in pain, fatigue and depression.

\section{Cancer}

Several forms of cancer have benefited from yoga including breast cancer, neck cancer and lymphoma. Breast cancer is the cancer that is most frequently diagnosed in women. And, although survival rates are continually increasing, breast cancer is often associated with chronic pain, fatigue and depression Cramer, Lauche, Klose, Lange, Langhorst \& Dobos [112]. In a randomized, controlled, single-blind trial, one group received a 10 -week yoga program and the other group received a 10-week follow-up program Eyigor, Uslu, Apaydin, Caramat \& Yesil [113]. Shoulder and arm pain intensity decreased significantly, and the benefit was surprisingly maintained at 2.5 months posttreatment. In a systematic review and meta-analysis on yoga for breast cancer studies, 24 studies with a total of 2166 participants were included Cramer et al. [112]. Seventeen studies comparing yoga versus no therapy provided moderate evidence for reduced pain, fatigue and sleep disturbances. In contrast, three studies that compared yoga versus exercise showed low-quality evidence for yoga effects. In a meta-analysis by another group, 17 studies met criteria that included 2183 patients with breast cancer Dong, Xie, Jing, Lin \& Tian [114]. Moderate evidence from this analysis suggested reduced fatigue.

\section{Neck cancer has also benefited from yoga}

In a randomized controlled study, participants were assigned to an eight-week Hatha yoga program and were compared to a waitlist - control group Adair, Murphy, Yarlagadda, Deng, Dietrich 
\& Ridner [115]. By the end of the two-month period, those who received yoga had significantly reduced pain and anxiety and increased shoulder range of motion. In a home-based yoga program for patients suffering from malignant lymphoma during chemotherapy, the positive yoga effects were decreased sleep problems, fatigue, depression and anxiety.

In a review of 29 randomized controlled trials on yoga for several kinds of cancer, fatigue was the most commonly measured outcome and was typically reduced following yoga Danhauer, Addington, Cohen, Sohl, Van Puymbroeck, Albinati et al. [116]. In addition, several randomized controlled trials showed that yoga improved biomarkers of stress including inflammation and immune function. The authors, nonetheless, suggested several directions for future research including standardizing the assessments, increasing the use of active control groups and objective measures and exploring effects of different types of yoga, the different components (movement, breathing and meditation), the dose and duration of the course of treatment.

\section{HIV}

HIV is another immune condition that has benefited from yoga. In a study on $60 \mathrm{HIV}$-positive individuals from rehabilitation centers, yoga participants experienced two months of yoga for one hour per day for five days a week Kuloor, Kumari \& Metri [117]. The yoga, once again, consisted of physical postures, breathing practices, and meditation. As compared to a waitlist control group, the yoga group experienced a greater reduction in fatigue, anxiety and depression and a greater improvement in well-being and quality of life. Unfortunately, as in most of the research on cancer, immune function was not measured. This is surprising given that several stress hormones and immune factors can currently be assayed in saliva samples such that the process is not invasive.

\section{Aging Conditions}

In this section, recent research is reviewed on yoga effects on conditions associated most frequently with aging. These include menopause, COPD, cardiovascular disease, stroke, Parkinson's and dementia. see (Table 7) for list on yoga effects on aging conditions.

Table 7: Adult yoga effects on aging conditions and first authors.

\begin{tabular}{|c|c|}
\hline Effects & First authors \\
\hline < vasomotor symptoms during menopause & Cramer 2018 \\
\hline $\begin{array}{c}\text { > forced expiratory volume at one second } \\
\text { in COPD }\end{array}$ & Li, Cramer 2019 \\
\hline$<$ inflammation in cardiovascular disease \\
\hline$>$ physical memory recovery after stroke & Guddeti \\
\hline$>$ Postural stability \& gait in Parkinson's & Adams, Van Puymbroek \\
\hline \multicolumn{2}{|c|}{$<$ falling } \\
\hline$>$ attention \& verbal memory & Brenes \\
\hline$>$ Posture control, flexibility in dementia & Green \\
\hline
\end{tabular}

\section{Menopause}

A recent systematic review and meta-analysis identified 13 randomized controlled trials with 1306 participants who experienced menopause Cramer, Peng \& Lauche [118]. Yoga, as compared with no treatment control groups, reduced menopausal symptoms including psychological, somatic, vasomotor and urogenital symptoms. However, as compared with exercise controls, the yoga group only experienced fewer vasomotor symptoms. The authors also noted that the effects were robust against selection bias but not against detection and attrition bias. But, importantly, no serious adverse events were reported.

\section{Chronic obstructive pulmonary disease (COPD)}

COPD is another condition that has apparently been the focus of many studies given that at least two systematic reviews and meta-analyses appeared in the recent literature. In the first of these, ten studies met inclusion criteria and showed greater improvements on several scales as well as the classical clinical pulmonary measure, namely FEV1 (forced expiratory volume at one second) Li, Liu, Ji, Xie \& Hou [119]. Another systematic review and meta-analysis supported these results based on 11 randomized controlled trials with a total of 586 patients Cramer, Haller, Klose, Ward, Chung \& Lauche [120]. In this case, improvements were noted in both exercise capacity as assessed by the six-minute walk test and pulmonary function, again as measured by FEV1. These were the only effects that were robust against methodological bias.

\section{Cardiovascular disease}

Cardiovascular disease continues to be the leading cause of morbidity and mortality around the world Guddeti, Dang, Williams \& Alla [121]. In a literature search on several databases including PubMed, Google scholar, Embase and Cochrane databases, yoga was noted to have favorable effects on stress, the cardiac autonomic nervous system, cardiovascular risk factors and systemic inflammation.

\section{Stroke}

In a systematic review of recent studies on yoga for acquired brain injury, yoga was specifically effective for stroke in terms of physical memory recovery even when yoga was compared to exercise control groups Silviera \& Smart [122]. The authors suggested that future research would benefit from having a common yoga protocol for stroke including frequency, length and duration of yoga as well as including psychophysiological measures and cultural factors as variables in the research.

\section{Parkinson's}

Parkinson's affects nearly 10 million people worldwide, leading to decreased mobility and balance that result in frequent falls that, in turn, lead to a loss of independence Adams, Van Puymbroeck, Walter, Hawkins, Schmid \& Sharpe [123]. In a randomized, wait-list-controlled study, yoga was given twice per 
week for two months Van Puymbroeck, Walter, Hawkins, Sharp, Woschkolup, et al. [124]. The yoga group experienced improved motor function, postural stability and gait as well as fewer falls. In a secondary analysis by the same group, body responsiveness was predictive of a decrease in Parkinson's-specific symptoms and gait velocity was predictive of an increase in balance and activity Adams et al. [123]

\section{Dementia}

Dementia has been the focus of two reviews in the recent literature on yoga. In a scoping review, eight studies suggested beneficial effects of yoga on cognitive function, particularly on attention and verbal memory Brenes, Sohl, Well, Befus, Campos [125].The positive effects on cognitive function may have been mediated by improved sleep and mood as well as increased neural connectivity. In a systematic review, the benefits of yoga included improved posture control, flexibility and relaxation, less falling and decreased anxiety and stress Green, Huynh, Broussard, Zunker, Matthews, et al. [94]. Several limitations of this literature include the lack of intervention details, variability of the frequency and duration of yoga as well as the components of the yoga interventions (postures, breathing and meditation). This research is also confounded by the variety of cognitive impairments including the different types of dementia and Alzheimer's disease.

\section{Special Samples}

In this section, research is reviewed on the use of yoga with special samples including veterans and prison inmates. Although most mental health and physical health conditions have associated comorbidities, adults of these special samples typically experience both mental health and physical health conditions and they are treated as a separate section in this review for that reason. see (Table 8) for list on yoga effects on special samples.

Table 8: Yoga effects on special samples including veterans and prison inmates.

\begin{tabular}{|c|c|}
\hline Effects & First authors \\
\hline \multicolumn{2}{|c|}{ Veterans } \\
\hline$<$ low back pain & Groessi, Highland \\
\hline$<$ PTSD symptoms & Cushing \\
\hline$<$ pain, depression, anxiety & Schulz-Meik \\
\hline \multicolumn{2}{|c|}{ Prison inmates } \\
\hline$<$ antisocial behavior & Kerekes \\
\hline < somatization symptoms & Sfendia \\
\hline$<$ depression \& anxiety & Bartels \\
\hline
\end{tabular}

\section{Veterans}

Research on veterans has focused on low back pain, posttraumatic stress disorder, depression and anxiety. Low back pain has been notably reduced in at least a couple studies on veterans. In one study, veterans were randomly assigned to yoga or waitlist control groups to engage in yoga classes (including home practice) twice weekly for 12 weeks Groessl, Liu, Chang, Wetherell, Bormann, et al. [126]. The yoga sessions were comprised of postures and breathing techniques. Surprisingly, low back pain was not only reduced by the end of the treatment period but also three months later, suggesting that the yoga group may have continued their home practice. In another study on low back pain in veterans, pain was again reduced in the yoga as compared with the control group, although no improvement was noted on physical functioning Highland, Schoomaker, Rojas, Suen, Ahmed, Zhang et al. [127].

Posttraumatic stress disorder was surprisingly the focus of only one study in the recent literature on yoga given the high incidence of posttraumatic stress disorder especially in veterans (9\% shortly after returning from deployment to $31 \%$ a year after deployment) Cushing, Braun, Alden C-layt \& Katz [128]. In this study, veterans participated in 60-minute weekly yoga sessions for six weeks using Vinyasa - style yoga. PTSD symptoms were decreased as evidenced by lower scores on the PTSD checklist - military version including hyperarousal, re-experiencing and avoidance. The veterans also reported decreased insomnia, depression and anxiety symptoms. Yoga has also been delivered to veterans via telehealth Schultz-Heik, Meyer, Mahoney, Stanton, Cho, Moore-Downing et al. [129]. Pain, depression and anxiety were reduced following this cost-effective form of yoga.

\section{Prison inmates}

Prison inmates have been noted to benefit from yoga including a reduction in risks associated with antisocial behavior, stress, depression and sleep problems. In one of three studies in the recent literature, a 10-week yoga group (one class a week) as compared to a control group (free of choice weekly physical activity) showed less antisocial behavior Kerekes, Brandstrom \& Nilsson [130]. In a separate report by the same research group, the weekly 90-minute yoga classes resulted in significantly less psychological stress and they had a positive effect on the obsessive-compulsive, paranoid ideation and somatization symptom factors on the Brief Symptom Inventory Sfendia, Malmstrom, Torstensson \& Kerekes [131]. In a study by a different research group, the weekly yoga program resulted in lower levels of depression, anxiety, and negative affect Bartels, Oxman \& Hopkins [132]. The inmates also reported improved flexibility, sleep and relaxation, pain reduction and improved mental well-being.

\section{Biomarkers}

This section includes summaries of research on physiological and biochemical biomarkers affected by yoga. Most of the recent yoga literature on physiological biomarkers has focused on heart rate variability and brain connectivity. Most of the research on biochemical biomarkers has been focused on cortisol and 
pro-inflammatory cytokines, especially IL-6. The research on biomarkers suggests potential underlying mechanisms for the yoga effects on different conditions.

\section{Physiological biomarkers}

The physiological biomarkers that have been affected by yoga according to the recent literature include skin conductance, respiration, blood pressure, vagal activity (heart rate variability), brain connectivity and gray matter volume. The recent inclusion of these measures in studies on yoga has not only provided confirmatory data for the self-reports on positive effects of yoga but has also helped further an understanding of potential underlying mechanisms for the effects of yoga. see (Table 9) for list on physiological biomarkers of yoga.

Table 9: Physiological biomarkers of yoga and first authors.

\begin{tabular}{|c|c|}
\hline Effects & First authors \\
\hline$<$ respiration & Cotton, Yamamoto-Morimoto \\
\hline < systolic blood pressure & Cramer 2018 \\
\hline $\begin{array}{c}<\text { systolic and diastolic blood } \\
\text { pressure }\end{array}$ & $\mathrm{Wu}$ \\
\hline $\begin{array}{c}>\text { heartrate variability (vagal } \\
\text { activity) }\end{array}$ & $\begin{array}{l}\text { Hayase,Vidyashree,Pradnya,Telles, } \\
\text { Christa, RivesGadbois,Zou }\end{array}$ \\
\hline$>$ functional connectivity in brain & Santaella \\
\hline > gray matter hippocampus & Gothe \\
\hline $\begin{array}{c}\text { > volume right amygdala \& left } \\
\text { hippocampus }\end{array}$ & Gotink \\
\hline
\end{tabular}

\section{Respiration}

In a study where yogis were compared to controls on their responses to warm and painfully hot stimuli, yogis breathed more slowly and deeply than controls Cotton, Low, Villemure \& Bushnell [133]. In another study, a yoga poses group was compared to a yoga poses plus breathing exercises group Yamamoto-Morimoto, Horibe, Takao \& Anami [134]. Respiratory function was measured by an auto spirometer. As might be expected, the addition of the breathing exercises resulted in improved respiratory function. In a study on cardio-respiratory function, adolescents were randomized to receive one hour of daily yoga training or physical exercise over a period of two months Satish, Rao, Manjunath, Amritanshu, Vivek, Shreeganesh et al. [35]. In this study, $\mathrm{VO}_{2}$ (the maximum amount of oxygen utilized during exercise) was assessed as a measure of aerobic endurance or cardiovascular fitness. There was a greater improvement in VO2 in the yoga group possibly because the exercise group experienced the exercise as too strenuous.

\section{Blood pressure}

Yoga has been used to lower blood pressure in hypertensive individuals in a couple studies in the recent literature. In one of these, a yoga poses group was compared to a breathing exercises group Cramer, Sellin, Schumann \& Dobos [135]. Surprisingly, only the breathing exercises group showed decreased systolic blood pressure. A systematic review and meta-analysis that yielded 49 qualified controlled trials on 3517 participants with high blood pressure, highlights the frequent use of yoga as an anti-hypertensive therapy $\mathrm{Wu}$, Johnson, Acabchuk, Chen, Lewis, et al. [136]. In these studies, yoga was practiced on average five sessions per week for 60 minutes per session for 13 weeks. On average, yoga elicited moderate reductions in both systolic and diastolic blood pressure. When yoga sessions occurred three times per week, those sessions that included breathing techniques and meditation elicited greater reductions as compared with those that did not. These data, once again, highlight the additional benefits of breathing techniques and meditation during yoga sessions.

\section{Heart rate variability (vagal activity)}

Heartrate variability has been measured in several yoga studies on a variety of conditions including pregnancy, autism, muscular dystrophy, low back pain and myocardial infarction. Invariably heart rate variability has increased. In a study on pregnant women, for example, heart rate variability during the night and during the late-night was higher in the yoga group Hayase, \& Shimada [137]. Nighttime sleep duration was also longer and salivary alpha-amylase levels (a stress hormone) also decreased. These results are likely inter-related.

In a randomized controlled trial on 62 patients with chronic low back pain, one group received yoga for three months while the other group received care as usual Telles, Bhardwaj AK, Gupta, Sharma, Monro \& Balkrishna [138]. The yoga group showed a significant decrease in the low-frequency power of heart rate variability and a significant increase in the high-frequency power of heart rate variability suggesting a shift in the autonomic balance toward greater vagal activity. In a randomized controlled trial on patients with post myocardial infarction, a 12-week yogabased program was given as an adjunct to standard care while the control group received standard care plus educational sessions Christa, Srivastava, Chandran, Jaryal, Yadav, Roy et al. [139]. At the end of the 12-week period, the yoga group had higher highfrequency power values suggesting an increase in heart rate variability (vagal activity).

Both narrative and systematic reviews on yoga effects on heart rate variability highlight the frequent use of this measure. In a narrative review, for example, 32 out of 61 articles met inclusion criteria Rivest-Gadbois, \& Boudrias [140]. These not only showed increased heart rate variability but also increased pain tolerance. In a systematic review and meta-analysis, 17 medium to high quality randomized controlled trials showed significantly beneficial effects on heart rate variability parameters including increased high-frequency power and decreased low frequency to high-frequency ratio Zou, Sasaki, Wei, Huang, Yeung, Neto et al. [141]. 


\section{Brain connectivity and gray matter volume}

The use of resting-state functional magnetic resonance imaging has enabled research on functional connectivity following yoga practice. In a study on elderly yoga practitioners as compared to yoga-naïve controls, the yoga practitioners practiced Hatha yoga twice a week for at least eight years Santaella, Balardin, Afonso, Giorjiani, Sato \& Lacerda [142]. The yoga group showed greater functional connectivity of the medial prefrontal cortex and the posterior cingulate cortex which would contribute to a healthier cognitive aging process.

In a study on gray matter volume in the hippocampus, thalamus and caudate nucleus, participants who were experienced yoga practitioners defined as having more than three years of regular yoga practice were compared to age and sex-matched controls without yoga experience Gothe, Hayes, Temali \& Damoiseaux [143]. The yoga practitioners had greater gray matter volume in the left hippocampus which is consistent with improved working memory following yoga practice. On the other hand, in another study on magnetic resonance scans in yoga practitioners, those who practiced yoga and meditation reported significantly more stress and more depressive symptoms that, in turn, were associated with significantly lower right amygdala volume and lower left hippocampal volume Gotink, Vernooij, Ikram, Niessen, Krestin, Hofman et al. [144]. These different right-left findings are difficult to interpret as are the inconsistent findings on brain volume.

\section{Biochemical Biomarkers}

Table 10: Biochemical biomarkers of yoga and first authors.

\begin{tabular}{|c|c|}
\hline Effects & First authors \\
\hline$<$ total cholesterol \& low-density lipoprotein & Azami \\
\hline$<$ whole blood viscosity & Shadiow \\
\hline$<$ cortisol & Ma \\
\hline \multicolumn{2}{|c|}{$>$ testosterone } \\
\hline$<$ cortisol \& immunoglobulin A \\
\hline$<$ pro-inflammatory marker (<IL-6)
\end{tabular}

The biochemical markers that have been assessed in the recent literature on yoga include cholesterol, whole blood viscosity, cortisol, testosterone and pro-inflammatory cytokines including IL-6, CRP and TNF. These markers have generally suggested lower stress and inflammation in yoga practitioners. They would likely interact with the physiological markers just discussed as, for example, the decrease in cortisol that accompanies increased vagal activity [1] see (Table 10) for biochemical biomarkers of yoga.

\section{Cholesterol}

In a study that assessed yoga effects on the lipid profile, yoga was conducted three times a week for 26 weeks Azami, HafeziAhmadi, YektaKooshali \& Qavam [145]. By the end of the study, both total cholesterol and low-density lipoprotein cholesterol were decreased. Surprisingly, no change was noted in high density lipoprotein as high- and low-density lipoprotein levels typically shift together.

\section{Whole blood viscosity}

Elevated whole blood viscosity has been linked to increased risk for cardiovascular disease Shadiow, Tarumi, Dhindsa \& Hunter [146]. In this study, yoga practitioners who had a minimum of three years consistent practice had significantly lower whole blood viscosity than sedentary, healthy adults.

\section{Cortisol}

Cortisol, which as a stress hormone has negative effects on immune function, has significantly decreased following yoga in at least two recent studies. In one of these, female patients with skeletal muscle pain syndrome were assigned to a yoga exercise group or a non-exercise control group (Ha 2015). After a 12-week study, the yoga group exhibited a greater decrease in cortisol levels. Cortisol has decreased even following one session of yoga Eda, Ito, Shimizu, Suzuki, Lee \& Akama [147]. In this study, cortisol decreased after 90 minutes of yoga stretching as did immunoglobulin A, and testosterone increased. Testosterone increases have often been associated with decreased cortisol and decreased cortisol is associated with enhanced immune function [1].

\section{Pro-inflammatory markers}

Decreased pro-inflammatory markers may also relate to decreased cortisol following yoga. Three different research groups have recently reported the reduction of pro-inflammatory markers following yoga, most frequently the cytokine IL-6. In a randomized controlled trial, a Hatha yoga group was compared to a health education control group Nugent, Brick, Armey, Tyrka, Ridout \& Uebelacker [148]. After a 10-week treatment period, plasma samples revealed a significant reduction in IL-6 in the yoga group. It's not clear why noninvasive saliva samples weren't assayed instead of blood samples in that study.

In another randomized controlled trial, a group of adults with metabolic syndrome were assigned to a 12-week yoga lifestyle intervention or a dietary intervention Yadav, Yadav, Khadgawat \& Pandey [149]. The yoga group not only showed a decrease in the pro-inflammatory marker IL -6 but also in a number of oxidative stress measures. A systematic review of the literature on yoga effects on inflammatory biomarkers yielded 16 studies that met criteria Djalilova, Schultz, Berger, Case, Kupzyk \& Ross [150]. The most common biomarkers measured were IL-6, C-reactive protein and tumor necrosis factor which were reduced following yoga. 
The authors mentioned the typical methodological problems of these studies including considerable variability in yoga types, components, frequency, session length, intervention duration and intensity.

\section{Methodological Limitations and Future Directions}

Most of the methodological limitations that were found in the earlier literature and discussed in the [1] review have continued to be limitations of the more recent research. In addition, the focus on clinical samples has introduced additional variability problems for the recent research. Several methodological problems relate to the research designs. First, the samples vary on the yoga experience of their participants, with some being virtual beginners and others being experienced yogis. Groups would need to be homogenous or carefully balanced on self-selection factors such as these. Experienced yogis would certainly be in better condition at baseline and more likely to practice yoga at home or at other classes between intervention sessions.

Secondly, many studies are not randomized, controlled trials. Some are single-arm, pre-post studies that lack a control group, some are mixed design (experimental versus control or waitlist control), and some are comparisons between active yoga versus inactive control groups such as educational groups. Often the comparisons between yoga and inactive control groups have favored the yoga groups, but when yoga is compared to an active group such as another form of exercise, the yoga and exercise often have equivalent effects. For example, in a study comparing yoga with tai chi and stretching, the groups were similar on exertion and pleasure Follador, Alves, Ferriera, Silva \& Silva [151].

But, in a systematic review and meta-analysis of randomized controlled trials, the yoga effects were superior to those of both the inactive controls (for example, wait list control, education booklets) and the active controls (for example, walking, chair aerobics) Sivaramakrishnan, Fitzsimons, Kelly, Ludwig, Mutrie, et al. [152]. This was true for physical variables including balance, lower body flexibility and lower limb strength as well as quality of life variables including depression, perceived mental health, perceived physical health, sleep quality and vitality. The more positive effects for yoga in this case may relate to the active control exercises being too rigorous or causing too much exertion. Random assignment is essential to avoid self-selection effects that would result in group differences at baseline, for example, in physical condition, that, in turn, would bias the outcomes. Systematic reviews and meta-analyses are now standard ways of grouping data but they usually require randomized controlled trials (RCTs) as one of their criteria. The meta-analyses cannot be performed if there are a limited number of RCTs that both meet criteria and are bias free. In addition, many of the RCTs have had such variable protocols that they could not be grouped for metaanalyses.

The variability in assessment protocols has also limited the grouping of RCTs for systematic reviews and meta-analyses. Self- reported stress, mood, pain and quality of life have been the most frequent measures but the self-report scales on these measures have varied across studies. Different self-report measures have been used for different conditions and in different cultures. In addition to lacking standardization of the less objective selfreport measures across studies, the gold standard measures for a condition have rarely been included, e.g. CD4 levels for HIV and natural killer cells for breast cancer.

Despite the absence of these convergent physical and physiological measures in many studies, these measures have been increasingly used in the recent literature as compared to that reviewed in 2016 [1]. For example, body mass index, blood pressure, heart rate variability, cortisol and pro-inflammatory cytokines (that are now relatively easy to assay from saliva samples) have been increasingly measured. Although those may be more objective measures, they would desirably be assessed together with the self-report measures that are frequently used and yoga logs (diaries) that are less frequently used Uebelacker, Feltus, Jones, Tremont \& Miller [153]. in order to have convergent data. Although most studies have used baseline and end-oftreatment assessments to determine yoga effects, some have conducted follow-up assessments to determine how sustainable the effects are. An example is a study that offered 60-minute yoga classes once a week for eight weeks that included all three components of yoga Lundt \& Jentschke [154]. A follow-up was conducted at six months after the end of yoga therapy. At that time, anxiety, depression and fatigue were significantly reduced compared to baseline, but they had increased during the followup period which is not surprising since the yoga sessions had been discontinued.

Another problem is that many different types of yoga, e.g. Ashtanga, Vinyasa, Iyengar and Restorative yoga, have been used for different conditions, making cross-study comparisons difficult. Some forms of yoga are considered more intense such as Iyengar yoga with its poses being held for longer durations and Ashtanga yoga with its continuous movements. The different yoga types need to be compared in future studies given that a specific form of yoga may be more effective for a specific condition. Some have attempted to quantify the different types on various dimensions such as breathwork and physical pose using a new scale they have developed called the Essential Properties of Yoga Questionnaire. This could be a very useful measure in comparing the different types of yoga for different conditions. The delivery of yoga sessions is also highly variable including individual versus group sessions and the dosage including the length of classes (20-90 minutes), the frequency of classes (daily, weekly) and the duration of the intervention (weeks, months).

Another confounding variable is that yoga is often an integrated practice of poses, breathing exercises and meditation. Although the poses predominate most yoga sessions, making yoga a type of physical exercise, the unique effects of the poses, 
breathing and meditation are unknown. Although yoga is thought to be a moderate intensity exercise, it may be less intense when it is combined with meditation resulting in lesser effects than those experienced by an active exercise group. However, both poses, and meditation have been noted to have parasympathetic effects [1]. Thus, combining them might have additive, positive effects. The poses, breathing and meditation may have synergistic effects that are critical to the yoga practice. For example, in a study on respiratory function, a group that did both poses and breathing exercises understandably had better respiration than a group that simply practiced poses. But the subcomponents of yoga classes have rarely been assessed for their individual effects. An exception was a study that assessed the most frequently researched components of yoga including physical postures, breathing methods and meditation Franklin \& Bentley [155]. In this comparison of the subcomponents, the poses were negatively correlated with psychological distress, anxiety and depression but by contrast, the correlations between the other yoga subcomponents and the symptoms scales were nonsignificant after accounting for exposure to life stressors. Additional studies are needed to determine which aspects or if all aspects are leading to the positive physical, physiological, cognitive and emotional effects being reported. But for studies to at least be replicable, more detailed descriptions of these components are needed in terms of the physical poses, breathing and meditation and the duration of each component. Oxygen consumption measures on each component might also indicate the exercise intensity of each component.

Similarly, comparisons are needed of the various poses. For example, in a study that used electromyography (EMG) to record muscle activity in the upper, middle and lower trapezius during 15 different yoga postures, the "locust arms forward" posture elicited the highest activity from the upper, middle and lower trapezius muscles. In contrast, the dancer's pose, reverse tabletop and warrior two postures demonstrated low activity of the scapular stabilizers. These are important data given that strength training of the scapular stabilizer muscles is the goal for shoulder rehabilitation. In another EMG study, activity was notably greater in the anterior tibialis and the gastrocnemius muscles for the tree pose, half-moon and warrior three highlighting the increased use of ankle musculature during the single limb yoga poses Kelley \& Romano [156].

The mode of delivery of yoga has rarely been assessed including comparisons between yoga class and individual yoga sessions and between groups that received yoga classes but also are encouraged to practice yoga at home. To encourage accessibility to yoga, some have suggested providing real-time interactive video called Teleyoga Mathersul \& Bayley [89]. In a randomized controlled trial on tele-yoga for hypertension, for example, two groups received yoga during three weeks of inpatient rehabilitation and then one group received tele-yoga for six weeks after discharge Sarah \& Claudia [157]. The tele-yoga group had significantly lower blood pressure at the follow-up assessment. As another example, women undergoing radiation or chemotherapy for breast cancer were given twelve 75-minute biweekly Internet-based multipoint video-conferenced yoga classes, and the women experienced less psychological distress Addington \& Danhauer [158-165].

Suggestions for future research include more frequent use of randomized controlled trials that compare yoga to other forms of exercise. Given that the benefits of yoga have been documented, it may be unethical to assign participants to inactive control groups. More studies are needed on physical, physiological and biochemical effects to complement the self-report data and additional potential underlying mechanism studies are needed. Research is also needed on duration of sessions. Yoga might be practiced more often if sessions were shorter and could be practiced at home. Nonetheless, the studies reviewed here highlight the therapeutic effects of yoga, a practice that is for that reason increasingly being called yoga therapy.

\section{Acknowledgements}

I would like to thank my collaborators, the individuals who participated in these studies and the research associates who assisted us. This research was supported by funding from Johnson and Johnson and from Massage Envy. Requests for reprints should be sent to Tiffany Field.

\section{References}

1. Field T (2016) Yoga Research Review. Complement Ther Clin Pract 24: 145-161.

2. Wang CC, Li K, Choudhury A, Gaylord S (2019) Trends in yoga, tai chi, and qigong use among US adults, 2002-2017. American Journal of Public Health 109(5): 755-761.

3. Cramer H, Ward L, Steel A, Lauche R, Dobos G, Zhang Y (2016) Prevalence, patterns, and predictors of yoga use: Results of a U.S. Nationally representative survey. Am J Prev Med 50: 230-235.

4. Patwardhan AR, Lloyd LW (2017) Decline in the use of medicalized yoga between 2002 and 2012 while the overall yoga use increased in the United States: A conundrum. J Evid Based Complementary Altern Med 22(4): 567-572.

5. Lauche R, Sibbritt D, Adams J, Cramer H (2019) Characteristics of yoga and meditation users among older Australian women- results from the 45 and up study. Complementary Therapies in Clinical Practices 35: 219-223.

6. Cramer H, Anheyer D, Saha FJ, Dobos G (2018) Yoga for posttraumatic stress disorder- A systematic review and meta-analysis. BMC Psychiatry 18(1): 72.

7. Sullivan M, Leach M, Snow J, Moonaz S (2017) The North American yoga therapy workforce survey. Complementary Therapeutic Medicine 31: 39-48.

8. Ross A, Touchton Leonard K, Kang L, Wallen G (2016) A national survey of yoga instructors and their delivery of yoga therapy. Int J Yoga Therap 26(1): 83-91.

9. Cramer H, Sundberg T, Schumann D, Leach MJ, Lauche R (2018) Differences between vegetarian and omnivorous practioners- Results of a nationally representative survey of US adult yoga practitioners Complement Ther Med 40: 48-52. 
10. Cramer H, Quinker D, Schumann D, Wardle J, Dobos G, et al. (2019) Adverse effects of yoga: A national cross-sectional survey. BMC Complementary and Alternative Medicine 19(1): 190.

11. Lee M, Huntoon EA, Sinaki M (2019) Soft tissue and bony injuries attributed to the practice of yoga: A biomechanical analysis and implications for management. Mayo Clinic Proceedings 94(3): 424431

12. Mears SC, Tackett SA, Elkins MC, Severin AC, Barnes SG, et al. (2019) Ankle motion in common yoga poses. Foot (Edinb) 39: 55-59.

13. Campo M, Shiyko MP, Kean, MB, Roberts L, Pappas E (2018) Musculoskeletal pain associated with recreational yoga participation: A prospective cohort study with 1-year follow-up. Journal of Bodywork and Movement Therapies 22: 418-423.

14. Cramer H, Lauche R, Anheyer D, Pilkington K, de Manincor M, et al. (2018) Yoga for anxiety: A systematic review and meta-analysis of randomized controlled trials. Depression and Anxiety 35(9): 830-843.

15. Launche R, Schumann D, Sibbritt D, Adams J, Cramer H (2017) Associations between yoga practice and joint problems: A crosssectional survey among 9151 Australian women. Rheumatology International 37(7): 1145-1148.

16. Kirca N, Pasinlioglu, T (2019) The effect of yoga on stress level in infertile women. Perspective Psychiatric Care 55(2): 319-327.

17. Darbandi S, Darbandi M, Khorram Khorshid HR, Sadeghi MR (2018) Yoga can improve assisted reproduction technology outcomes in couples with infertility. Alternative Therapeutic Health in Medicine 24(4): 50-55

18. Ng QX, Venkatanarayanan N, Loke W, Yeo WS, Lim DY, et al. (2019) A meta-analysis of the effectiveness of yoga-based interventions for maternal depression during pregnancy. Complementary Therapy in Clinical Practices 34: 8-12.

19. Jahdi F, Sheikhan F, Haghani H, Sharifi B, Ghaseminejad A, et al (2017) Yoga during pregnancy: The effects on labor pain and delivery outcomes (A randomized controlled trial). Complement Ther Clin Pract 27: 1-4.

20. Chethana B, Raghunandan C, Saili A, Mondfal S, Saxena P (2018) Prenatal yoga: Effects on alleviation of labor pain and birth outcomes. J Altern Complement Med.

21. Field T, Diego M, Medina L, Delgado J, Hernandez A (2012) Yoga and Massage Therapy Reduce Prenatal Depression and Prematurity. J Bodyw Mov Ther 16: 204-209.

22. Jarraya S, Wagner M, Jarraya M, Engel FA (2019) 12 weeks of kindergarten-based yoga practice increases visual attention, visual-motor precision and decreases behavior of inattention and hyperactivity in 5-year-old children. Front Psychol 10: 790-796.

23. Gulati K, Sharma SK, Telles S, Balkrishna A (2019) Self-esteem and performance in attentional tasks in school children after $41 / 2$ months of yoga. Int J Yoga 12(2): 158-161.

24. Nanthakumar C (2018) The benefits of yoga in children. Journal of Integrated Medicine 16: 14-19.

25. Vidyashree HM, Maheshkumar K, Sundareswaran L, Sakthivel G, Partheeban PK, et al. (2018) Effect of Yoga intervention on short-term heart rate variability in children with autism spectrum disorder. Int J Yoga 12(1): 73-77.

26. Pradnya D, Nalini A, Nagarathna R, Raju TR, Sendhilkumar R, et al (2019) Effects of yoga as an add-on therapy in the modulation of heart rate variability in children with Duchenne Muscular Dystrophy. Int J Yoga 12: 55-61.
27. Halliwell E, Jarman H, Tylka TL, Slater A (2018) Evaluating the impact of a brief yoga intervention on preadolescents' body image and mood. Body Image 27: 196-201.

28. Hainsworth KR, Liu XC, Simpson PM, Swartz AM, Linneman N, et al. (2018) A pilot study of lyengar yoga for pediatric obesity: Effects on gait and emotional functioning. Children (Basel 5(7) pii: E92.

29. Pandya S (2019) Yoga education program for reducing drug deprendency and promoting better asthma control for chronic asthmatic children: A multicity experiment. Global Pediatric Health 6: $2333794 X 19837455$.

30. McNamara C, Johnson M, Read L, Vander Velden H, Thygeson M, et al. (2016) Yoga therapy in children with cystic fibrosis decreases immediate anxiety and joint pain. Evidence Based Complementary and Alternative Medicine.

31. Kanhere SV, Bagadia DR, Phadke VD, Mukherjee PS (2018) Yoga in children with epilepsy: A randomized controlled trial. J Pediatr Neurosci 13(4): 410-415.

32. Kwasky AN, Serowoky ML (2018) Yoga to enhance self-efficacy: An Intervention for at-risk youth. Archives of Psychiatric Nursing 32: 8285.

33. Choukse A, Ram A, Nagendra HR (2019) Effect of residential yoga camp on psychosocial fitness of adolescents. Int J Yoga 12: 139-145.

34. Vhavle SP, Rao RM, Manjunath NK (2019) Comparison of yoga versus physical exercise on executive function, attention, and working memory in adolescent schoolchildren: A randomized controlled trial. Int J Yoga 12(2): 172-173.

35. Satish V, Rao RM, Manjunath NK, Amritanshu R, Vivek U, Shreeganesh HR, et al. (2018) Yoga versus physical exercise for cardio-respiratory fitness in adolescent school children: A randomized controlled trial. International Journal of Adolescent Medical Health.

36. Arruda JM, Bogetz AL, Vellanki S, Wren A, Yeh AM (2018) Yoga as an adjuct therapy for adolescents with inflammatory bowel disease: A pilot clinical trial. Complement Ther Med 41: 99-104.

37. Evans S, Seidman LC, Lung K, Sternlieb B, Zeltzer LK (2018) Yoga for teens with irritable bowel syndrome: Results from a mixed-methods pilot study. Holist Nurs Pract 32(5): 253-260.

38. Kishida M, Mogle J, Elavsky S (2019) The daily influences of yoga on relational outcomes off of the mat. Int J Yoga 12(2): 103-113.

39. Trent NL, Borden S, Miraglia M, Pasalis E, Dusek JA, et al. (2019) Improvements in psychological and occupational well-being in a pragmatic controlled trial of a yoga-based program for professionals. Journal of Alternative and Complementary Medicine 25(6): 593-605.

40. Blades R, Mac Fadyen D (2019) The psycho-linguistic effects of yoga: A lexical analysis of shifts in positivity, agency, and creativity. International Journal of Yoga 12: 91-95.

41. Telles S, Gupta RK, Kumar A, Pal DK, Tyagi D, et al. (2019) Mental wellbeing, quality of life, and perception of chronic illness in yogaexperienced compared with yoga-naïve patients. Medical Science Monitor Basic Research 25: 153-163.

42. Tulloch A, Bombell H, Dean C, Tiedemann A (2018) Yoga-based exercise improves health-related quality of life and mental well-being in older people: A systematic review of randomized controlled trials. Age and Ageing 47(4): 537-544.

43. Domingues RB (2018) Modern postural yoga as a mental health promoting tool: A systematic review. Complement Ther Clin Pract 31: 248-255. 
44. Maddux RE, Daukantaité D, Tellhed U (2018) The effects of yoga on stress and psychological health among employees: An 8- and 16-week intervention study. Anxiety Stress \& Coping 31(2): 121-134.

45. Cocchiara RA, Peruzzo M, Mannocci A, Ottolenghi L, Villari P, et al. (2019) The use of yoga to manage stress and burnout in healthcare workers: A systematic review. J Clin Med 8(3).

46. Puerto Valencia LM, Weber A, Spegel H, Bögle R, Selmani A, et al (2019) Yoga in the workplace and health outcomes: A systematic review. Occupational Medicine 69(3): 195-203.

47. Shohani M, Badfar G, Nasirkandy MP, Kaikhavani S, Rahmati S, et al. (2018) The effect of yoga on stress, anxiety, and depression in women. International Journal of Preventative Medicine 9: 19-21.

48. Field T, Diego M, Hernandez Reif M, Schanberg S, Kuhn C, et al. (2003) Pregnancy Anxiety and Comorbid Depression and Anger Effects on the Fetus and Neonate. Depress Anxiety 17(3): 140-151.

49. Saeed SA, Cunningham K, Bloch RM (2019) Depression and anxiety disorders: Benefits of exercise, yoga, and meditation. American Family Physician 99(10): 620-627.

50. Vollbehr NK, Bartels-Velthuis AA, Nauta MH, Castelein S, Steenhuis LA, et al. (2018) Hatha yoga for acute, chronic and/or treatment-resistant mood and anxiety disorders: A systematic review and meta-analysis. PLoS One 13(10): e0204925.

51. Nguyen-Feng VN, Clark CJ, Butler ME (2018) Yoga as an intervention for psychological symptoms following trauma: A systematic review and quantitative synthesis, Psychological Services 16(3): 513-523.

52. Tolahunase MR, Sagar R, DadaR (2018) 5-HTTLPR and MTHFR 677C>T polymorphisms and response to yoga-based lifestyle intervention in major depressive disorder: A randomized active-controlled trial. Indian Journal of Psychiatry 60(4): 410-426.

53. Bukar NK, Eberhardt LM, Davidson J (2019) East meets west in psychiatry: Yoga as adjunct therapy for management of anxiety. Archives of Psychiatric Nursing 33: 371-376.

54. Kelly UA, Evans DD, Baker H, Noggle Taylor J (2018) Determining Psychoneuroimmunology markers of yoga as an intervention for persons diagnosed with PTSD: A systematic review. Biological Research for Nursing 20(3): 343-351.

55. Jeffries ER, Zvolensky MJ, Buckner JD (2018) The acute impact of hatha yoga on craving among smokers attempting to reduce or quit. Nicotine and Tobacco Research.

56. Kuppili PP, Parmar A, Gupta A, Balhara YPS (2018) Role of yoga in management of substance-use disorders: A narrative review. J Neurosci Rural Pract 9(1): 117-122.

57. Köksoy S, Eti CM, Karataş M, Vayisoglu Y (2018) The effects of yoga in patients suffering from subjective tinnitus. Int Arch Otorhinolaryngol 22(1): 9-13

58. Kongkaew C, Lertsinthai P, Jampachaisri K, Mongkhon P, Meesomperm P, et al (2018) The effects of Thai yoga on physical fitness: A metaanalysis randomizing controlled trials. J Altern Complement Med 24(6): 541-551.

59. Neumark-Sztainer D, Watts AW, Rydell S (2018). Yoga and body image: How do young adults practicing yoga describe its impact on their body image? Body Image 27: 156-168.

60. Telles S, Sharma SK, Singh A, Kala N, Upadhyay V, et al. (2019) Quality of life in yoga experienced and yoga naïve Asian Indian adults with obesity. Journal of Obesity.

61. Pryor JL, Christensen B, Jackson CGR, Moore-Reed S (2019) Metabolic demands of yoga at varying tempos and compared with walking. J Phys Act Health 16(7): 575-580.
62. Karlsen KE, Vrabel K, Bratland-Sanda S, Ulleberg P, Benum K (2018) Effect of yoga in the treatment of eating disorders: A single-blinded randomized controlled trial with 6-months follow-up. International Journal of Yoga 11(2): 166-169.

63. Domingues RB, Carmo C (2019) Disordered eating behaviors and correlates in yoga practioners: A systematic review. Eat Weight Disord 24(6): 1015-10214

64. Schmid AA, Van Puymbroeck M, Fruhauf CA, Bair MJ, Portz JD (2019) Yoga improves occupational performance, depression, and daily activities for people with chronic pain. Work 63(2): 181-189.

65. Sutar R, Yadav S, Desai G (2016) Yoga intervention and functional pain syndromes: A selective review. International Review of Psychiatry 28(3): 316-322.

66. Monson AL, Chismark AM, Cooper BR, Krenik-Matejcek TM (2017) Effects of yoga on musculoskeletal pain. Journal of Dental Hygeine 91(2): 15-22.

67. Hartfiel N, Clarke G, Havenhand J, Phillips C, Edwards RT (2017) Costeffectiveness of yoga for managing musculoskeletal conditions in the workplace. Occup Med (Lond) 67(9): 687-695.

68. Sharma VM, Manjunath NK, Nagendra HR, Ertsey C (2018) Combination of Ayurveda and yoga therapy reduces pain intensity and improves quality of life in patients with migraine headache. Complementary Therapies in Clinical Practices 32: 85-91.

69. Allende S, Anandan A, Lauche R, Kramer H (2018) Effect of yoga on chronic non-specific neck pain: An unconditional growth model. Complement Ther Med 40: 237-242.

70. Kim SD (2018) Effects of yogic exercise on nonspecific neck pain in university students. Complementary Therapies in Clinical Practice 31: 338-342.

71. Dunleavy K, Kava K, Goldberg A, Malek MH, Talley SA, et al. (2016) Comparative effectiveness of pilates and yoga group exercise interventions for chronic mechanical neck pain: Quasi-randomized parallel controlled study. Physiotherapy 102(3): 236-242.

72. Kim SD (2016) Effects of yoga on chronic neck pain: A systemic review of randomized controlled trials. J Phys Ther Sci 28(7): 2171-2174.

73. Cramer H, Klose P, Brinkhaus B, Michalsen A, Dobos G (2017) Effects of yoga on chronic neck pain: A systematic review and meta-analysis. Clin Rehabil 31: 1457-1465.

74. Li Y, Li S, Jiang J, Yuan S (2019) Effects of yoga on patients with chronic nonspecific neck pain: A PRISMA systematic review and meta-analysis. Medicine (Baltimore) 98(8): e14649.

75. Chang DG, Holt JA, Sklar M, Groessl EJ (2016) Yoga as a treatment for chronic low back pain: A systematic review of the literature. J Orthop Rheumatol 3(1): 1-8.

76. Patil NJ, Nagarathna R, Tekur P, Patil DN, Nagendra HR, et al. (2015) Designing, validation, and feasibility of integrated yoga therapy module for chronic low back pain. Int J Yoga 8(2): 103-108.

77. Telles S, Bhardwaj AK, Gupta RK, Sharma SK, Monro R (2016) A randomized controlled trial to assess pain and magnetic resonance imaging-based (MRI-based) structural spine changes in low back pain patients after yoga practice. Medical Science Monitor 22: 3228-3247.

78. Kuvačić G, Fratini P, Padulo J, Antonio DI, De Giorgio A (2018) Effectiveness of yoga and educational intervention on disability, anxiety, depression, and pain in people with CLBP: A randomized controlled trial. Complementary Therapies in Clinical Practices 31: 262-267.

79. Patil NJ, Nagaratna R, Tekur P, Manohar PV, Bhargav H (2018) A randomized trial comparing effect of yoga and exercises on quality of 
life in among nursing population with chronic low back pain. Int J Yoga 11(3): 208-214.

80. Saper RB, Lemaster C, Delitto A, Sherman KJ, Herman PM, et al. (2017) Yoga, physical therapy, or education for chronic low back pain: A randomized noninferiority trial. Annals of Internal Medicine 167(2): 85-94.

81. Neyaz O, Sumila L, Nanda S, Wadhwa S (2019) Effectiveness of Hatha yoga versus conventional therapeutic exercises for chronic nonspecific low-back pain. Journal of Alternative \& Complementary Medicine 25(9): 938-945.

82. Whitehead A, Gould Fogerite S (2017) Yoga treatment for non-specific low back pain (2017) Explore (NY) 13(4): 281-284.

83. Wieland LS, Skoetz N, Pilkington K, Vempati R, D Adamo CR, et al. (2017) Yoga treatment for chronic non-specific low back pain. Cochrane Database Systematic Review 1: CD010671.

84. Goode AP, Coeytaux RR, McDuffie J, Duan-Porter W, Sharma P, et al. (2016) An evidence maps of yoga for low back pain. Complementary Therapies in Therapeutic Medicine 25: 170- 177.

85. Cheung C, Justice C, Peden Mc Alpine C (2015) Yoga adherence in older women six months post-osteoarthritis intervention. Global Advances in Health and Medicine 4(3): 16-23.

86. Park J, McCaffrey R, Newman D, Liehr P, Ouslander JG (2017) A pilot randomized controlled trial of the effects of chair yoga on pain and physical function among community-dwelling older adults with lower extremity osteoarthritis. Journal of American Geriatric Society 65(3): 592-597.

87. McCaffrey R, Park J, Newman D (2017) Chair yoga: Feasibility and sustainability study with older community-dwelling adults with osteoarthritis. Holistic Nursing Practices 31(3): 148-157.

88. Cheung C, Wyman JF, Bronas, U, Mc Carthy, T Rudser K, et al. (2017) Managing knee osteoarthritis with yoga or aerobic/strengthening exercise programs in older adults: A pilot randomized controlled trial. Rheumatol Int 37(3): 389-398.

89. Mathersul DC, Mahoney LA, Bayley PJ (2018) Tele-yoga for chronic pain: Current status and future directions. Global Advances in Health and Medicine 7: 21649561a118766011.

90. Kuntz AB, Chopp-Hurley JN, Brenneman EC, Karampatos S, Wiebenga EG, et al. (2018) Efficacy of a biomechanically-based yoga exercise program in knee osteoarthritis: A randomized controlled trial. PLoS One 13(4): e0195653.

91. Cheung C, Park J, Wyman JF (2016) Effects of yoga on symptoms, physical function, and psychosocial outcomes in adults with osteoarthritis: A focused review. Am J Phys Med Rehabil 95(2): 139151.

92. Kan L, Zhang J, Yang Y, Wang P (2016) The effects of yoga on pain, mobility, and quality of life in patients with knee osteoarthritis: A systematic review. Evidence Based Complementary and Alternative Medicine. Evid Based Complement Alternat Med.

93. Wang Y, Lu S, Wang R, Jiang P, Rao F. et al. (2018). Integrative effect of yoga practice in patients with knee arthritis: A PRISMA-compliant meta-analysis. Medicine (Baltimore 97(31): e11742.

94. Green E, Huynh A, Broussard L, Zunker B, Matthews J, et al. (2019) Systematic review of yoga and balance: Effect on adults with neuromuscular impairment. American Journal of Occupational Therapy 73(1): 7301205150p1-7301205150p11.

95. Jayawardena R, Ranasinghe P, Chathuranga T, Atapattu PM, Misra A (2018) The benefits of yoga practice compared to physical exercise in the management of type 2 Diabetes Mellitus: A systematic review and meta-analysis. Diabetes Metabolism Syndrome 12(5): 795-805.
96. Schmid AA, Atler KE, Malcolm MP, Grimm LA, Klinedinst TC, et al. (2018) Yoga Improves quality of life and fall risk factors in a sample of people with chronic pain and type 2 diabetes. Complementary Therapies in Clinical Practice 31: 369-373.

97. Mondal S, Kundu B, Saha S (2018) Yoga as a therapeutic intervention for the management of type 2 Diabetes Mellitus. Int J Yoga Therap 11(2): 129-138.

98. Sharma P, Poojary G, Dwivedi SN, Deepak KK (2015) Effect of yogabased intervention in patients with inflammatory bowel disease. Int J Yoga Therap 25(1): 101-112.

99. Schumann D, Anheyer D, Lauche R, Dobos G, Langhorst J (2016) Effect of yoga in the therapy of irritable bowel syndrome: A systematic review. Clinical Gastroenterology and Hepatology 14(12): 1720-1731.

100. Ghaffarilaleh G, Ghaffarilaleh V, Sanamno Z, Kamalifard M, Alibaf L (2018) Effects of yoga on quality of sleep of women with premenstrual syndrome. Alternative Therapy in Health Medicine 25(5): 40-47.

101. Ghaffarilaleh G, Ghaffarilaleh V, Sanamno Z, Kamalifard M (2019) Yoga positively affected depression and blood pressure in women with premenstrual syndrome in a randomized controlled clinical trial. Complement Ther Clin Pract 34: 87-92.

102. Tsai SY (2016) Effect of yoga exercise on premenstrual symptoms among female employees in Taiwan. Int J Environ Res Public Health 13(7). pii: E721.

103. Yang NY, Kim SD (2016) Effects of a yoga program on menstrual cramps and menstrual distress in undergraduate students with primary dysmenorrhea: A single-blind, randomized controlled trial. Journal of Alternative and Complementary Medicine 22(9): 732-738.

104. Yonglitthipagon P, Muansiangsai S, Wongkhumngern W, Donpunha W, Chanavirut R, et al. (2017) Effect of yoga on the menstrual pain, physical fitness, and quality of life of young women with primary dysmenorrhea. Journal of Bodywork and Movement Therapies 21(4): 840-846.

105. McGovern CE, Cheung C (2018) Yoga and quality of life in women with primary dysmenorrhea: A systematic review. J Midwifery Womens Health 63(4): 470-482.

106. Saxena R, Gupta M, Shankar N, Jain S, Saxena A (2017) Effects of yogic intervention on pain scores and quality of life in females with chronic pelvic pain. International Journal of Yoga 10(1): 9-15.

107. Huang AJ, Rowen TS, Abercrombie P, Subak LL, Schembri M, et al. (2017) Development and feasibility of a group-based therapeutic yoga program for women with chronic pelvic pain. Pain Medicine 18(10): 1864-1872.

108. Kinser PA, Pauli J, Jallo N, Shall M, Karst K, et al. (2017) Physical activity and yoga-based approaches for pregnancy-related low back and pelvic pain. J Obstet Gynecol Neonatal Nurs 46(3): 334-346.

109. Hasanpour Dehkordi A (2016) Influence of yoga and aerobics exercise on fatigue, pain and psychosocial status in patients with multiple sclerosis: A randomized trial. Journal of Sports Medicine and Physical Fitness 56(11): 1417-1422.

110. Hasanpour-Dehkordi A, Jivad N, Solati K (2016) Effects of yoga on physiological indices, anxiety and social functioning in multiple sclerosis patients: A randomized trial. Journal of Clinical Diagnostic Research 10(6): VC01-VC05.

111. Kahraman T, Ozdogar AT, Yigit P, Hosgel I, Mehdiyev Z, et al. (2018) Feasibility of a 6-month yoga program to improve the physical and psychosocial status of persons with multiple sclerosis. Explore (NY) 14(1): 36-43.

112. Cramer H, Lauche R, Klose P, Lange S, Langhorst J, et al. (2017) Yoga for improving health-related quality of life, mental health and 
cancer-related symptoms in women diagnosed with breast cancer Cochrane Database Syst Rev.

113. Eyigor S, Uslu R, Apaydin S, Caramat I, Yesil H (2018) Can yoga have any effect on shoulder and arm pain and quality of life in patients with breast cancer? A randomized, controlled, single-blind trial. Complement Ther Clin Pract 32: 40-45.

114. Dong B, Xie C, Jing X, Lin L, Tian L (2019) Yoga has a solid effect on cancer-related fatigue in patients with breast cancer: A metaanalysis. Breast Cancer Res Treat 177(1): 5-16.

115. Adair M, Murphy B, Yarlagadda S, Deng J, Dietrich MS, et al. (2018) Feasibility and preliminary efficacy of tailored yoga in survivors of head and neck cancer: A pilot study. Integr Cancer Ther 17(3): 744784 .

116. Danhauer SC, Addington EL, Cohen L, Sohl SJ, Van Puymbroeck M, et al. (2019) Yoga for symptom management in oncology: A review of the evidence base and future directions for research. Cancer 125(12): 1979-1989.

117. Kuloor A, Kumari S, Metri K (2019) Impact of yoga on psychopathologies and quality of life in persons with HIV: A randomized controlled study. Journal of Bodywork and Movement Therapies 23(2): 278-283.

118. Cramer H, Peng W, Lauche R (2018) Yoga for menopausal symptomsA systematic review and meta-analysis. Maturitas 109: 13-25.

119. Li C, Liu Y, Ji Y, Xie L, Hou Z (2018) Efficacy of yoga training in chronic obstructive pulmonary disease patients: A systematic review and meta-analysis. Complementary Therapies in Clinical Practices 30 33-37.

120. Cramer H, Haller H, Klose P, Ward L, Chung VC, et al. (2019) The risks and benefits of yoga for patients with chronic obstructive pulmonary disease: A systematic review and meta-analysis. Clinical Rehabilitation 33(13): 1847-1862.

121. Guddeti RR, Dang G, Williams MA, Alla VM (2018) Role of yoga in cardiac disease and rehabilitation. Journal of Cardiopulmonary Rehabilitation and Prevention.

122. Silveira K, Smart CM (2019) Cognitive, Physical, and psychological benefits of yoga for acquired brain injuries: A systematic review of recent findings. Neuropsychological Rehabilitation 21: 1-20.

123. Adams EV, Van Puymbroeck M, Walter A, Hawkins BL, Schmid AA, et al. (2019) Predictors of functional improvements after therapeutic yoga intervention for people with Parkinson's Disease. Int J Yoga Therap.

124. Van Puymbroeck M, Walter AA, Hawkins BL, Sharp JL, Woschkolup $\mathrm{K}$, et al. (2018) Functional improvements in Parkinson's Disease following a randomized trial of yoga. Evidence based Complementary Alternative Medicine.

125. Brenes GA, Sohl S, Well RE, Befus D, Campos CL, et al. (2019) The effects of yoga on patients with mild cognitive impairment and dementia: A scoping review. American Journal of Geriatric Psychiatry 27: 188-197.

126. Groessl EJ, Liu L, Chang DG, Wetherell JL, Bormann JE, et al. (2017) Yoga for military veterans with chronic low back pain: A randomized clinical trial. American Journal of Preventative Medicine 53(5): 599608.

127. Highland KB, Schoomaker A, Rojas W, Suen J, Ahmed A, et al. (2018) Benefits of the restorative exercise and strength training for operational resilience and excellence yoga program for chronic low back pain in service members: A pilot randomized controlled trial. Archives of Physical Medicine and Rehabilitation 99(1): 91-98.
128. Cushing RE, Braun KL, Alden C, layt SW, Katz AR (2018) Militarytailored yoga for veterans with post-traumatic stress disorder Military Medicine 183(5-6): e223-e231.

129. Schulz-Heik RJ, Meyer H, Mahoney L, Stanton MV, Cho RH, et al. (2017) Results from a clinical yoga program for veterans: Yoga via telehealth provides comparable satisfaction and health improvements to inperson yoga. BMC Complementary and Alternative Medicine 17(1): 191-198.

130. Kerekes N, Brändström S, Nilsson T (2019) Imprisoning yoga: Yoga practice may increase the character maturity of male prison inmates. Frontiers in Psychiatry 10: 401- 406.

131. Sfendla A, Malmström P, Torstensson S, Kerekes N (2018) Yoga practice reduces the psychological distress levels of prison inmates. Front Psychiatry 9: 401-407.

132. Bartels L, Oxman LN, Hopkins A (2019) I would just feel really relaxed and at peace: Findings from a pilot prison yoga program in Australia. Int J Offender Ther Comp Criminol 63(15-16): 2531-2549.

133. Cotton VA, Low LA, Villemure C, Bushnell MC (2018) Unique autonomic responses to pain in yoga practitioners. Psychosom Med 80: 791-798.

134. Yamamoto-Morimoto K, Horibe S, Takao R, Anami K (2019) Positive effects of yoga on physical and respiratory functions in healthy inactive middle-aged people. International Journal of Yoga 12(1): 62-67.

135. Cramer H, Sellin C, Schumann D, Dobos G (2018) Yoga in arterial hypertension. Dtsch Arztebl International 115: 833-839.

136. Wu Y, Johnson BT, Acabchuk RL, Chen S, Lewis HK, et al. (2019) Yoga as antihypertensive lifestyle therapy: A systematic review and metaanalysis. Mayo Clinic Proceedings 94(3): 432-446.

137. Hayase M, Shimada M (2018) Effects of maternity yoga on the autonomic nervous system during pregnancy. Journal of Obstetrics and Gynecology Research 44(10): 1887-1895.

138. Telles S, Sharma SK, Gupta RK, Bhardwaj AK, Balkrishna A (2016) Heart rate variability in chronic low back pain patients randomized to yoga or standard care. BMC Complementary Alternative Medicine 16(1): 271-279.

139. Christa E, Srivastava P, Chandran DS, Jaryal AK, Yadav RK, et al. 2019) Effect of yoga-based cardiac rehabilitation on heart rate variability: Randomized control trial in patients post-MI. International Journal of Yoga Therapy 29(1): 43-50.

140. Rivest-Gadbois E, Boudrias MH (2019) What are the known effects of yoga on the brain in relation to motor performances, body awareness and pain? A narrative review. Complementary Therapies in Medicine 44: $129-142$.

141. Zou L, Sasaki JE, Wei GX, Huang T, Yeung AS, et al. (2018) Effects of mind-body exercises (tai chi/yoga) on heart rate variability parameters and perceived stress: A systematic review with metaanalysis of randomized controlled trials. Journal of Clinical Medicine, $7(11)$. pii: E404.

142. Santaella DF, Balardin JB, Afonso RF, Giorjiani GM, Sato JR (2019) Greater anteroposterior default mode network functional connectivity in long-term elderly yoga practitioners. Frontiers in Aging Neuroscience 11: 151-158.

143. Gothe NP, Hayes JM, Temali C, Damoiseaux JS (2018) Difference in brain structure and function among yoga practitioners and controls. Front Integr Neurosci 22: 12:26.

144. Gotink RA, Vernooij MW, Ikram MA, Niessen WJ, Krestin GP, et al (2018) Meditation and yoga practice are associated with smaller 
right amydala volume: The Rotterdam study. Brain Imaging Behavior 12(6): 1631-1639.

145. Azami M, Hafezi Ahmadi MR, Yekta Kooshali MH, Qavam S (2019) Effect of yoga on lipid profile and C-reactive protein in women. Int J Prev Med 10: 81.

146. Shadiow J, Tarumi T, Dhindsa M, Hunter SD (2019) A comparison of blood viscosity and hematocrit levels between yoga practitioners and sedentary adults. International Journal of Exercise Science 12(2): 425-432.

147. Eda N, Ito H, Shimizu S, Suzuki S, Lee E, et al. (2018) Yoga stretching for improving salivary immune function and mental stress in middleaged and older adults. Journal of Women Aging 30(3): 227-241.

148. Nugent NR, Brick L, Armey MF, Tyrka AR, Ridout KK, et al. (2019) Benefits of yoga on IL-6: Findings from a randomized controlled trial of yoga for depression. Behavioral Medicine 29: 1-10.

149. Yadav R, Yadav RK, Khadgawat R, Pandey RM (2018) Comparative efficacy of a 12 week yoga-based lifestyle intervention and dietary intervention on adipokines, inflammation, and oxidative stress in adults with metabolic syndrome: A randomized controlled trial. Translational Behavioral Medicine.

150. Djalilova DM, Schultz PS, Berger AM, Case AJ, Kupzyk KA, et al. (2019) Impact of yoga on inflammatory biomarkers: A systematic review. Biol Res Nurs 21(2): 198-209.

151. Follador L, Alves RC, Ferriera SDS, Silva AC, Silva SGD (2019) Perceived exertion and affect from tai chi, yoga, and stretching classes for elderly women. Perceptual and Motor Skills 126(2): 223-240.

152. Sivaramakrishnan D, Fitzsimons C, Kelly P, Ludwig K, Mutrie N, et al (2019) The effects of yoga compared to active controls on physical function and health related quality of life in older adults- Systematic review and meta-analysis of randomized controlled trials. Int J Behav Nutr Phys Act 16(1): 30-33.

153. Uebelacker LA, Feltus S, Jones R, Tremont GN, Miller IW (2019) Weekly assessment of number of yoga classes and amount of yoga home practice: Agreement with daily diaries. Complementary Therapies in Medicine 43: 227-231.

154. Lundt A, Jentschke E (2019) Long-term changes of symptoms of anxiety, depression, and fatigue in cancer patients 6 months after the end of yoga therapy. Integr Cancer Ther 18: 1534735418822096.
155. Franklin RA, Butler MP, Bentley JA (2018) The physical postures of yoga practices may protect against depressive symptoms, even as life stressors increase: A moderation analysis. Psychol Health Med 23(7): 870-879.

156. Kelley KK, Giannico K, Lesnett G, Romano A (2019) A comparison of EMG output of four lower extremity muscles during selected yoga postures. J Bodyw Mov Ther 23(2): 329-333.

157. Sarah S, Wolfgang MB, Claudia P (2019) Effect of telerehabilitation on long-term adherence to yoga as an antihypertensive lifestyle intervention: Results of a randomized controlled trial. Complement Ther Clin Pract 35: 148-153.

158. Addington EL, Sohl SJ, Tooze JA, Danhauer SC (2018) Convenient and Live Movement (CALM) for women undergoing breast cancer treatment: Challenges and recommendations for internet-based yoga research. Complement Ther Med 37: 77-79.

159. Campo M, Shiyko MP, Kean, MB, Roberts L, Pappas E (2018) Musculoskeletal pain associated with recreational yoga participation: A prospective cohort study with 1-year follow-up. Journal of Bodywork and Movement Therapies 22: 418-423.

160. Chopp Hurley JN, Prophet C, Thistle B, Police J, Maly MR (2018) Scapular muscle activity during static yoga postures. J Orthop Sports Phys Ther 48(6): 504-509.

161. Cramer H, Ostermann T, Dobos G (2018) Injuries and other adverse events associated with yoga practice: A systematic review of epidemiological studies. Journal of Science and Medicine in Sport 21(2): 147-154.

162. Eyigor S, Uslu R, Apaydin S, Caramat I, Yesil H (2018) Can yoga have any effect on shoulder and arm pain and quality of life in patients with breast cancer? A randomized, controlled, single-blind trial. Complement Ther Clin Pract 32: 40-45.

163. Field T (2019) Social Touch, CT Touch and Massage Therapy. Developmental Review 51: 123-145.

164. Ha MS, Baek YH, Kim JW, Kim DY (2015) Effects of yoga exercise on maximum oxygen uptake, cortisol level, and creatine kinase myocardial bond activity in female patients with skeletal muscle pain syndrome. Journal of Physical Therapy Science 27(5): 1451-1453.

165. Park CL, Elwy AR, Maiya M, Sarkin AJ, Riley KE, et al (2018) The essential properties of yoga questionnaire (EPYQ): Psychometric properties. International Journal of Yoga Therapy 28(1): 23-38.

\begin{tabular}{l} 
Your next submission with Juniper Publishers \\
will reach you the below assets \\
- Quality Editorial service \\
- Swift Peer Review \\
- Reprints availability \\
- E-prints Service \\
- Manuscript Podcast for convenient understanding \\
- Global attainment for your research \\
- Manuscript accessibility in different formats \\
( Pdf, E-pub, Full Text, Audio) \\
- Unceasing customer service \\
Track the below URL for one-step submission \\
https://juniperpublishers.com/online-submission.php \\
\hline
\end{tabular}

